\title{
Fusarium verticillioides SGE1 Is Required for Full Virulence and Regulates Expression of Protein Effector and Secondary Metabolite Biosynthetic Genes
}

\author{
Daren W. Brown, Mark Busman, and Robert H. Proctor \\ Bacterial Foodborne Pathogens and Mycology Research, United States Department of Agriculture-Agricultural Research \\ Service National Center for Agricultural Utilization Research (NCAUR), 1815 N. University St., Peoria, IL 61604, U.S.A.
}

Submitted 27 September 2013. Accepted 7 April 2014.

\begin{abstract}
The transition from one lifestyle to another in some fungi is initiated by a single orthologous gene, $S G E 1$, that regulates markedly different genes in different fungi. Despite these differences, many of the regulated genes encode effector proteins or proteins involved in the synthesis of secondary metabolites (SM), both of which can contribute to pathogenicity. Fusarium verticillioides is both an endophyte and a pathogen of maize and can grow as a saprophyte on dead plant material. During growth on live maize plants, the fungus can synthesize a number of toxic SM, including fumonisins, fusarins, and fusaric acid, that can contaminate kernels and kernel-based food and feed. In this study, the role of $F$. verticillioides $S G E 1$ in pathogenicity and secondary metabolism was examined by gene deletion analysis and transcriptomics. $S G E 1$ is not required for vegetative growth or conidiation but is required for wild-type pathogenicity and affects synthesis of multiple SM, including fumonisins and fusarins. Induced expression of $S G E 1$ enhanced or reduced expression of hundreds of genes, including numerous putative effector genes that could contribute to growth in planta; genes encoding cell surface proteins; gene clusters required for synthesis of fusarins, bikaverin, and an unknown metabolite; as well as the gene encoding the fumonisin cluster transcriptional activator. Together, our results indicate that $S G E 1$ has a role in global regulation of transcription in $F$. verticillioides that impacts but is not absolutely required for secondary metabolism and pathogenicity on maize.
\end{abstract}

Fungi are an ecologically diverse group of organisms that can live on a variety of substrates in different environments and can exhibit vastly different lifestyles. Animal- and plant-

Mention of trade names or commercial products in this article is solely for the purpose of providing specific information and does not imply recommendation or endorsement by the United States Department of Agriculture (USDA). USDA is an equal opportunity provider and employer.

Corresponding author: D. W. Brown; Telephone: +1.309.681.6230; Fax: +1.309.681.6672; E-mail: daren.brown@ars.usda.gov

* The $e$-Xtra logo stands for "electronic extra" and indicates that 15 supplementary tables and one supplementary figure are published online.

This article is in the public domain and not copyrightable. It may be freely reprinted with customary crediting of the source. The American Phytopathological Society, 2014. pathogenic fungi often exist among the normal microflora of their hosts without causing disease symptoms. Wor1/Ryp1 is a transcriptional regulator governing transition between different lifestyles that also requires morphological transition and was first characterized in the human pathogens Candida albicans and Histoplasma capsulatum (Huang et al. 2006; Nguyen and Sil 2008). In $C$. albicans, Wor 1 controls transition between white and opaque cell types, which grow preferentially at different host infection sites, by affecting the expression of several hundred genes (Huang et al. 2006; Zordan et al. 2007). In $H$. capsulatum, the Wor1 homolog, Ryp1, controls transition from saprophytic filamentous growth to pathogenic yeast-like growth at $37^{\circ} \mathrm{C}$ (Nguyen and Sil 2008).

Wor1/Rhyp1 orthologs have been identified in the plantpathogenic fungi Fusarium graminearum (Fgp1) (Jonkers et al. 2012), F. oxysporum (Sge1) (Michielse et al. 2009b), Botrytis cinerea (Reg1) (Michielse et al. 2011), and Verticillium dahliae (Sge1) (Santhanam and Thomma 2013). Inactivation of genes encoding these homologous proteins (collectively referred to hereafter as Sge1) results in some phenotypes common to all four of these plant-pathogenic fungi as well as some phenotypes that are unique to one or two species. In all species examined, $S G E 1$-inactivation mutants exhibit reduced levels of pathogenicity on their respective hosts. However, in the different fungi, the reduction may result from loss of different virulence factors such as effector proteins or phytotoxic secondary metabolites (SM) that require Sge1 for their production. In $F$. oxysporum, reduced virulence was attributed to reduced expression of effectors (Michielse et al. 2009b). Effectors are secreted proteins and include small cysteine-rich peptides, cell-wall-degrading enzymes, and necrosis-inducing proteins (de Jonge et al. 2011). Effectors and SM can alter host physiology, likely by suppressing defenses, and thereby allow pathogens to grow in the host (de Jonge et al. 2011; Rafiqi et al. 2012). The F. oxysporum Sge1 homolog controls expression of numerous effectors including secreted-in-xylem (SIX) proteins, some of which are required for pathogenicity on tomato (Houterman et al. 2007, 2009; Michielse et al. 2009b; Takken and Rep 2010; van der Does et al. 2008). Although the $V$. dahliae Sge1 homolog was shown to positively regulate some effectors and negatively regulate others, a direct role in disease has not yet been established (Santhanam and Thomma 2013). In F. graminearum and $B$. cinerea, reduced virulence was attributed to loss of production of phytotoxic sequiterpenoids (Jonkers et al. 2012; Michielse et al. 2011). The F. graminearum Sge1 homolog, Fgp1, affects the expression of a gene cluster responsible for 
synthesis of trichothecene toxins, which contribute to the ability of this fungus to cause wheat head blight (Jonkers et al. 2012). As with Wor1/Ryp1 in the human pathogens, Sge1 homologs in plant-pathogenic fungi play a critical role in pathogenicity, perhaps by enabling fungi to grow in planta (Michielse et al. 2009b).

F. verticillioides is one of the most common maize-associated fungi in most regions of the world where this crop is grown. The fungus can be readily isolated from maize and maize products, as well as from dead plant material in the soil (Burgess 1981; Nelson 1992). Although it occurs most frequently as an endophyte, causing no obvious detrimental effects, $F$. verticillioides can also occur in maize as a pathogen, causing seed, root, stalk, and ear rot as well as seedling blight (Bacon et al. 2001; Murillo et al. 1999; Oren et al. 2003). The biological, environmental, and physical factors that affect transition of $F$. verticillioides between its saprophytic, endophytic, and pathogenic growth phases are poorly understood.

During growth in maize, $F$. verticillioides can produce SM, including some that are toxic to animals (e.g., mycotoxins), that can contaminate maize and maize-based products. Fumonisin mycotoxins are a particular concern, because their consumption is epidemiologically associated with esophageal cancer and neural tube defects in humans and has been demonstrated to cause diseases in horses, swine, and experimental rodents (Glenn 2007; Marasas et al. 2004; Morgavi and Riley 2007). The contamination of grain and grain products with mycotoxins has a significant impact on health costs and international trade estimated to be in the hundreds of millions of dollars each year ( $\mathrm{Wu}$ 2007). The role of fumonisins in the ecology of $F$. verticillioides is not clear. In some studies, fumonisin production contributed to the pathogenicity of $F$. verticillioides on maize seedlings (Glenn et al. 2008; Myung et al. 2011) but, in others, it did not (Desjardins et al. 2007). In addition, results from two studies provided no evidence for a role for fumonisin production in the ability of $F$. verticillioides to cause maize ear rot (Desjardins and Proctor 2007; Desjardins et al. 2002). However, because most SM exhibit some level of toxicity or other biological activities, they have the potential to play a role in saprophytism by suppressing growth of competing microorganisms or in endophytism by suppressing host defenses or growth of other endophytes. It is also possible that toxic SM could contribute to pathogenicity of a microorganism by suppressing growth of competing pathogens.

Based on the previously characterized critical roles of Sge1 homologs in SM production and virulence in plant-pathogenic fungi, we sought to determine whether Sge1 functions in SM production, pathogenicity, and gene regulation in $F$. verticillioides. Our results show that Sge1 is required for wild-type levels of virulence on maize seedlings and wild-type fumonisin production in culture. Transcriptomic analysis indicates that Sge1 affects the transcription of hundreds of genes, including genes associated with the synthesis of at least four SM families and numerous potential effector genes.

\section{RESULTS}

\section{In silico characterization of $\boldsymbol{F}$. verticillioides $S G E 1$.}

Previously, Jonkers and associates (2012) noted that the $F$. verticillioides gene model FVEG_09150 in the Broad Institute's Comparative Fusarium Database is predicted to encode an Sge1-like protein. Here, further characterization of FVEG_ 09150 indicated that it consists of a 987-bp, intronless coding region predicted to encode a 328-amino-acid (aa) protein. Like other Sge1 homologs (Jonkers et al. 2012), the predicted FVEG_09150 protein consists of a conserved amino-terminal region of approximately 220 aa and a variable carboxy termi- nal region of approximately 120 aa. The conserved region of FVEG_09150 shares 80 and 82\% amino acid sequence identity with the corresponding region of the Sge1 homologs in $F$. oxysporum (FoSge1) and F. fujikuroi (FfSge1; FFUJ_07864) respectively, while the conserved regions of FoSge1 and FfSge 1 share $89 \%$ identity. In contrast, the FVEG_09150 variable region shares 42 and $58 \%$ amino acid sequence identity with the corresponding region in FoSge1 and FfSge1, respectively, while the variable regions in FoSge1 and FfSge1 share $30 \%$ identity. FVEG_09510 is represented by accession TC33724 in the $F$. verticillioides Gene Index, an expressed sequence tag (EST) database at the Dana Farber Cancer Institute (Brown et al. 2005). TC33724 comprises 20 EST derived from six cDNA libraries, each generated from a different growth condition. Seventeen of the EST were derived from $F$. verticillioides grown in a liquid medium for different periods of time, two were from growth on cracked maize kernel medium, and one was from germinating conidia (Brown et al. 2005). No FVEG_09510/TC33724 EST were recovered from growth of the fungus on excised maize seedling roots and shoots. Analysis of transcriptomic data (Gene Expression Omnibus [GEO] accession number GSE16900) derived from $F$. verticillioides grown for $24,48,72$, and $96 \mathrm{~h}$ in a liquid fumonisin production medium revealed significant expression of FVEG_09150 at all timepoints. The high levels of sequence identity of the predicted FVEG_09510 protein and Sge1 homologs in other fusaria indicate that the predicted FVEG_09510 protein is the $F$. verticillioides Sge1 homolog. The EST and microarray data indicate that the corresponding gene, $S G E 1$, is expressed under multiple growth conditions.

\section{SGE1 deletion, complementation, and inducible expression.}

In order to examine its function, the $F$. verticillioides SGE1 was deleted, complemented, and subjected to inducible expression. For deletion, we used the split-marker gene deletion strategy combined with protoplast-mediated transformation (Catlett et al. 2002; Turgeon et al. 1987) to replace the SGE1 coding region with the hygromycin $\mathrm{B}$ resistance gene, $H y g B$ (Catlett et al. 2002; Turgeon et al. 1987) (Fig. 1A). For complementation, a fragment that included the SGE1 promoter, coding, and $3^{\prime}$ flanking regions was cloned adjacent to the geneticin resistance gene GenR to create plasmid pSGE1C (Bluhm et al. 2008). For inducible expression, we first generated plasmid pXLN2P::SGE1, in which the SGE1 coding and $3^{\prime}$ flanking regions were fused to the promoter region of the $F$. verticillioides xylanase gene XLN2 (FVEG_12502) (Fig. 2). Protoplast-mediated co-transformation was then used to introduce pXLN2P::SGE1 and plasmid pKS-Gen, which carries GenR into the sgel mutant $\triangle \mathrm{SGE} 1.12$.

A polymerase chain reaction (PCR) screen identified four independent transformants that lacked the $S G E$ lcoding region, a result that was confirmed by Southern analysis in two of the transformants: $\Delta \mathrm{SGE} 1.6$ and $\Delta \mathrm{SGE} 1.12$ (Fig. $1 \mathrm{~B}$ and $\mathrm{C}$ ). Following transformation of the sgel mutant $\Delta$ SGE1.12 with the complementation vector pSGE1C, complemented mutants were identified by a PCR assay that amplified the entire SGE1 coding region using primers specific to the $S G E 1$ promoter and terminator regions (data not shown). Three complemented mutants (strains $\Delta$ SGE1.12AB1, $\Delta$ SGE1.12AB15, and $\Delta$ SGE1.12AB19) identified in this analysis were selected for further study. Following transformation of the sgel mutant $\triangle \mathrm{SGE} 1.12$ with the inducible expression vector pXLN2P::SGE1, transformants with the $X L N 2$ promoter-SGE1 fusion construct were identified by PCR using primers specific to the XLN2 promoter and $S G E 1$ coding sequence (data not shown). In transformants carrying the fusion construct, expression of SGE1 should be induced by xylose. We confirmed xylose-induced expression 
in one transformant, strain GMTcp-272, by growing it in SYP medium (100 mM sorbitol, $0.25 \%$ peptone, and $0.075 \%$ yeast extract) and adding $50 \mathrm{mM}$ xylose after $18 \mathrm{~h}$ of incubation. The SYP medium was chosen to help minimize general transcription; thus allowing for easier visualization of new transcripts induced by the appearance of Sge1. Using reverse-transcriptase (RT)-PCR with $S G E 1$-specific primers 1836 and 1838 , we observed $S G E 1$ expression in strain GMTcp-272 at 5, 15, and $30 \mathrm{~min}$ after the addition of xylose (Fig. 2B). In contrast, no $S G E 1$ expression was observed in the sgel progenitor strain $\triangle \mathrm{SGE} 1.12$ (Fig. 2B) or in the wild-type progenitor of the sgel mutant (data not shown). Thus, addition of xylose to cultures of the SGE1 inducible-expression strain GMTcp-272 generated high levels of expression of $S G E 1$ within 5 min.

We also assessed whether addition of xylose to cultures of GMTcp-272 resulted in expression of a gene that might be regulated by SGE1. In F. oxysporum, the gene SIX2 is regulated by SGE1 (Michielse et al. 2009b; Rep et al. 2002). The gene model FVEG_03307 in the Fusarium Comparative Database is likely the $F$. verticillioides SIX2 ortholog because it shares $68.2 \%$ deduced amino acid identity with $F$. oxysporum SIX2. RT-PCR analysis with the FVEG_03307-specific primers 1881 and 1882 revealed expression of FVEG_03307 30 min after addition of xylose to cultures of GMTcp-272 (Fig. 2C). In contrast, no expression of FVEG_03307 was observed following addition of xylose to cultures of the sgel mutant or wild type. These results provide evidence that $S G E 1$ functions similarly in $F$. verticillioides and $F$. oxysporum with respect to regulation of SIX2 and, therefore, potentially other genes as well.

\section{Phenotypic characterization \\ of mutants and inducible-expression strain.}

Vegetative growth and conidiation of two independent sgel mutants (strains $\Delta$ SGE1.6 and $\Delta$ SGE1.12) did not differ significantly from the wild type in two liquid media (GYP [2\% glucose, $1 \%$ peptone, and $0.3 \%$ yeast extract $]$ and CMC $[15 \mathrm{~g}$ of carboxymethylcellulose], $1 \mathrm{~g}$ of $\mathrm{NH}_{4} \mathrm{NO}_{3}, 1 \mathrm{~g}$ of $\mathrm{KH}_{2} \mathrm{PO}_{4}$, $0.5 \mathrm{~g}$ of $\mathrm{MgSO}_{4} \cdot 7 \mathrm{H}_{2} \mathrm{O}$, and $1 \mathrm{~g}$ of yeast extract per liter]) and on two solid media (V8 and sGYP [GYP plus $1.5 \%$ agar]) (Supplementary Table S1; Supplementary Fig. S1).

The effect of the deletion of $S G E 1$ on pathogenicity of $F$. verticillioides was examined using a standard maize seedling assay (Glenn et al. 2008; Larson et al. 2011) with 'Silver Queen' and plant height as a measure of disease severity (Fig. $3)$. In the assay, the virulence of both sgel mutants was significantly reduced compared with the wild type (Table 1). Complementation of the sgel mutant $\Delta$ SGE1.12 restored virulence to wild-type levels (Table 2; Fig. 3). Although virulence of the $S G E 1$ inducible-expression strain was not restored to wildtype levels, in the presence of supplemental xylose, it exhib-
A Split marker-based deletion of SGE1

Transforming DNA
(2 amplicons)
Wild-type
SGE1

Deleted SGE1
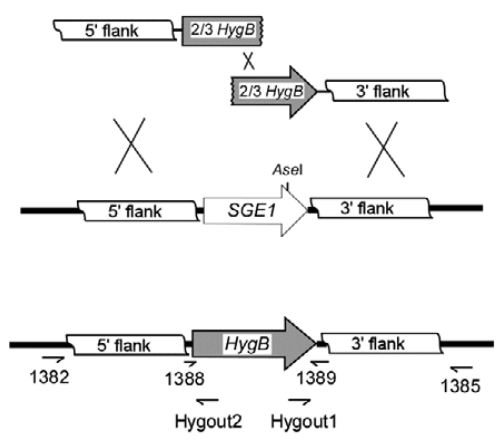

\section{B PCR analysis}

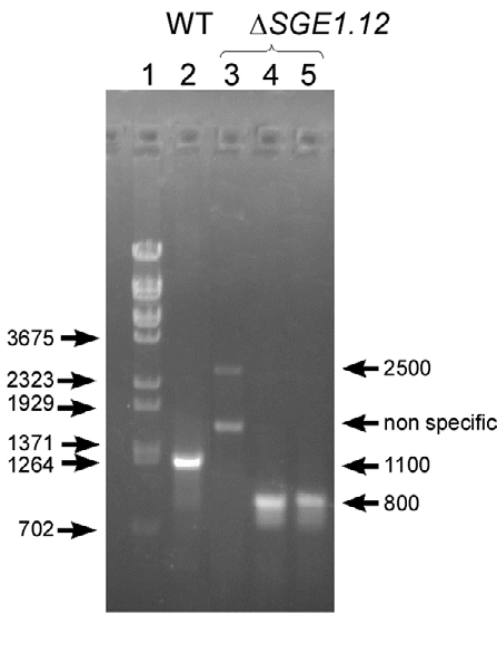

Southern analysis

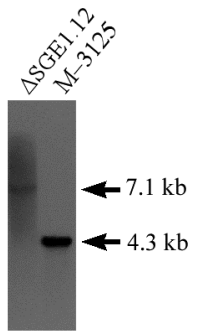

Fig. 1. SGE1 deletion in Fusarium verticillioides. A, Split-marker approach used for deletion, showing the three homologous recombination events required for replacement of the $S G E 1$ coding region (white arrow) with the hygromycin resistance gene, $H y g B$ (gray arrow). Small black arrows labeled as 1382 , 1385, 1388, 1389, Hygout1, or Hygout2 indicate positions of primers used for polymerase chain reaction (PCR) analysis of transformants. B, PCR strategy used to determine whether $S G E 1$ was deleted, showing results from a representative transformant, strain $\triangle$ SGE1.12. In PCR, primer combinations 1382Hygout2 and 1385-Hygout1 yielded approximately 800-bp amplicons that confirm integration of transforming DNA into the SGE1 locus. These primer combinations do not yield an amplicon from the wild type or transformants with an intact SGE1. In addition, primers 1388 and 1389 , which are complementary to the region immediately outside of the targeted deletion, confirmed the presence of $\mathrm{Hyg} B$ by amplifying a 2,500-bp product from $\mathrm{sge} 1$ mutants but a 1,100bp product from the wild type or transformants with an intact SGE1. Lane 1, DNA ladder; lanes 2 and 3, primer 1388-1389 amplicons; lane 4, primer 1382Hygout2 amplicon; lane 5, primer 1385-Hygout1 amplicon. Sizes of amplicons are indicated in base pairs to right of gel image. C, Southern analysis of $\triangle \mathrm{SGE1.12}$. Genomic DNA was digested with AseI and probed with a fragment of DNA downstream from the predicted SGE1 start codon (e.g., the left flank). $S G E 1$ sequence includes an AseI restriction site; thus, transformants digested with this enzyme, lacking SGE1, and probed would display a DNA band larger $(7.1 \mathrm{~kb})$ than the band observed in the wild type $(4.3 \mathrm{~kb})$. 
ited significantly higher levels of virulence than the sge1 mutant $(\triangle \mathrm{SGE} 1.12)$ from which it was derived (Supplementary Table S2). The addition of xylose had no effect on virulence of the wild type or any effect on median height of uninoculated plants based on Dunnett's test at $P \leq 0.05$.

Pathogenicity of $\triangle \mathrm{SGE} 1.12$ and three complemented sgel mutants was also examined using a modified maize seedling assay in which pieces of maize stalk colonized by a single $F$. verticillioides strain were placed into the soil $24 \mathrm{~h}$ prior to sowing. Visual inspection did not reveal any differences in colonization of the stalk pieces by the sgel mutant and complemented mutant strains. In this experiment, two of the three complemented strains exhibited significantly higher levels of virulence than the sgel mutant progenitor strain $\Delta$ SGE1.12 (Table 2). The lack of discernible differences in growth in and on laboratory media and in maize stalks of sgel mutants and the wild type or complemented mutants indicates that a functional $S G E 1$ is not essential for the saprophytic lifestyle of $F$. verticillioides. In contrast, the reduction in maize seedling disease caused by the mutant relative to the wild type and complemented mutants indicates that $S G E 1$ contributes to the pathogenic lifestyle of the fungus.
We also examined the effect of $S G E 1$ deletion on production of three $F$. verticillioides SM (fumonisins, fusaric acid, and fusarins), using a previously described cracked maize kernel medium assay and liquid chromatography tandem mass spectrometry (LC-MS/MS) analysis (Brown et al. 2012). Both sgel mutant strains $(\Delta$ SGE1.6 and $\Delta$ SGE1.12) exhibited reduced production of fumonisins and increased production of fusarins. On average, the levels of fumonisins and fusarins produced by the mutants were 39 and $140 \%$, respectively, of the levels produced by the wild-type progenitor strain (Table 3). Fusaric acid production was variable in sgel mutants, ranging from 48 to $109 \%$ of wild-type levels. Fumonisin production increased 4.4-fold in two SGE1-complemented strains and twofold in the third strain relative to the sgel mutant progenitor (Table 3). Fumonisin production also increased 4.8-fold in the SGE1 inducible-expression strain in the absence of exogenous xylose (Table 3). This result was not unexpected because the maize pericarp contains hemicellulose which includes xylan, a polymer of xylose (Nghiem et al. 2011). The failure of fumonisin production to be restored to wild-type levels in the three complemented mutants could result from integration of the complementation construct into a genomic region that did not

\section{A Construction of $S G E 1$ expression vector}

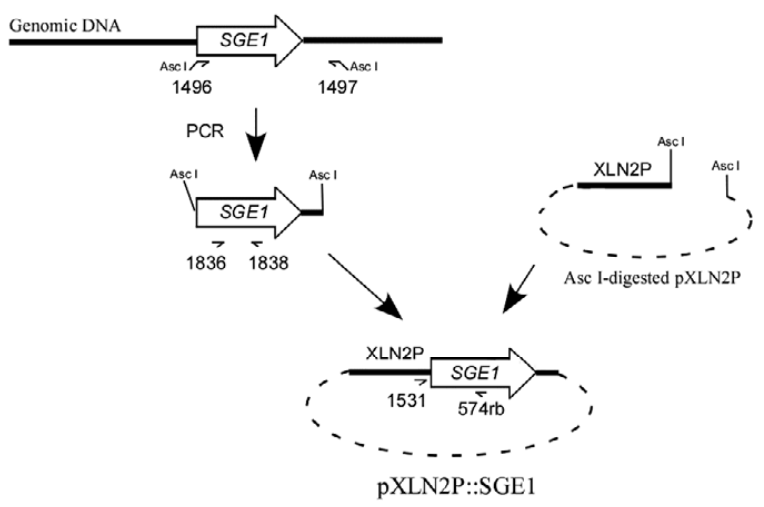

B Xylose induced expression of $S G E 1$

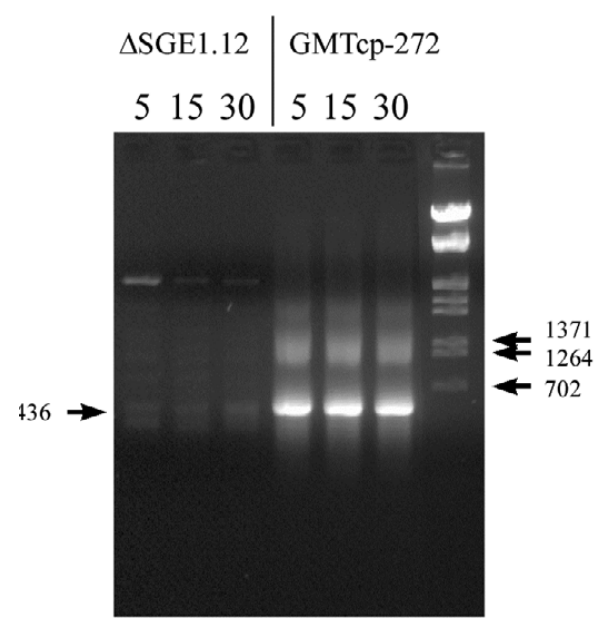

C SGE1 induced SIX2 expression

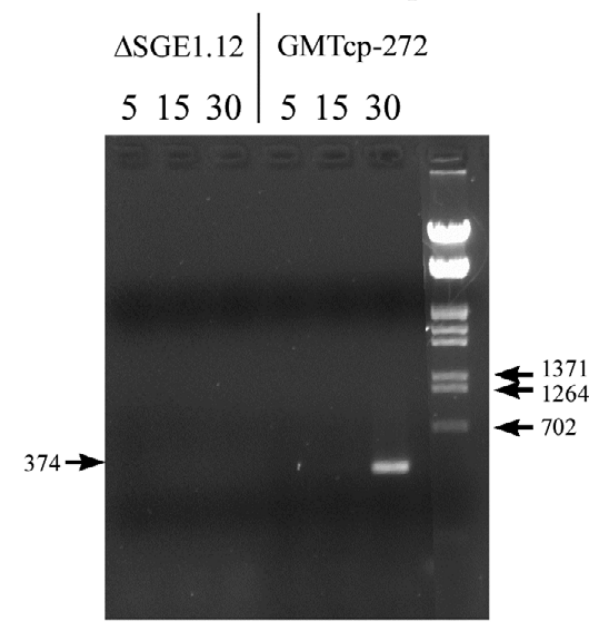

Fig. 2. Induced expression of $S G E 1$ and putative effector gene $F v S I X 2$. A, Construction of $S G E 1$ inducible-expression vector pXLN2P::SGE1 by ligating the $S G E 1$ coding region (white arrow) and its $3^{\prime}$ flanking region immediately downstream of the XLN2 promoter region in plasmid pXLN2P. B, Reverse-transcriptase polymerase chain reaction (RT-PCR) analysis of induced expression of SGE1. sge1 mutant strain $\triangle$ SGE1.12 and SGE1 inducible-expression strain GMTcp-272 were grown in liquid SYP medium; after $18 \mathrm{~h}$ of incubation, cultures were induced by addition of $50 \mathrm{mM}$ xylose; and mycelia were harvested for RNA preparation 5, 15, and 30 min later. RT-PCR with $S G E 1$-specific primers 1836 and 1838 yielded the predicted 436-bp amplicon at each time point from the $S G E 1$ inducible-expression strain. No PCR product was amplified from the sge1 mutant ( $\triangle$ SGE1.12). C, Induction of the FvSIX2 expression in SGE1 inducible-expression strain. FvSIX2-specific primers 1881 and 1882 amplified the predicted 374-bp product from only the SGE1 inducible-expression strain. Gel lanes: lane L, DNA ladder; lanes 5, 15, and 30 indicate time (in minutes) after induction by addition of xylose. Sizes of amplicons are indicated in base pairs to the right of gel image. 
allow $S G E 1$ to function as effectively as the native gene in the wild-type strain.

$S G E 1$ positively and negatively regulates multiple genes.

To begin to elucidate the genetic pathways through which $S G E 1$ affects pathogenicity and secondary metabolism, we conducted a transcriptomic analysis of the sgel mutant, SGE1 inducible-expression strain, and their wild-type progenitor. Each strain was grown in liquid SYP medium for $18 \mathrm{~h}$, at which time $50 \mathrm{mM}$ xylose was added. RNA was extracted from cultures $30 \mathrm{~min}$ later and subjected to microarray analysis. In pairwise comparisons of the three strains, 142 to 1,388 genes exhibited a greater than twofold change in expression, either an increase or a decrease, as a result of $S G E 1$ deletion or induced expression (Supplementary Tables S3, S4, S5, and S6; Table 4). In some cases, changes in expression were greater than eightfold. The data also indicate that SGE1 affects expression of more genes in positive manner (i.e. increases expression) than in a negative manner (i.e. decreases expression). For example, 168 genes were greater than fourfold more highly expressed in the wild type than in the sgel mutant, whereas only 34 genes were greater than fourfold more highly expressed in the mutant than the wild type (Table 4). Likewise, 435 genes were greater than fourfold more highly expressed in the $S G E 1$ inducible-expression strain than in the sgel mutant, whereas only 117 genes were greater than fourfold more highly ex-

Table 1. Pathogenicity of sge1 mutants of Fusarium verticillioides in maize seedlings assay

\begin{tabular}{llcl}
\hline Strain & Genotype & $\begin{array}{c}\text { Number of } \\
\text { plants }^{\mathbf{a}}\end{array}$ & $\begin{array}{c}\text { Mean height } \\
(\mathbf{c m})^{\mathbf{b}}\end{array}$ \\
\hline None & .. & 28 & $36.4 \pm 6.7 * *$ \\
M-3125 & Wild type & 26 & $24.2 \pm 6.6$ \\
$\Delta$ SGE1.6 & sge1 Mutant & 22 & $31.7 \pm 6.8^{* *}$ \\
$\Delta$ SGE1.12 & sge1 Mutant & 26 & $34.8 \pm 5.3 * *$ \\
\hline
\end{tabular}

a At the time disease was assessed, the number of living maize seedlings above $10 \mathrm{~cm}$ that resulted from 30 seeds sown in three pots (10 seeds/ pot).

${ }^{b}$ Height of each seedling was determined by measuring distance from the soil surface to the distal end of the longest leaf. Maize seedlings infected with M-3125 had significantly lower mean height than strains followed by ** based on Dunnett's test at $P \leq 0.05$. pressed in the mutant relative to the inducible-expression strain (Table 4).

There was considerable overlap between the groups of genes that exhibited higher or lower levels of expression in the wild type and sgel mutant compared with the SGE1 inducibleexpression mutant. Of the 135 genes that were $>8$-fold more highly expressed in the $S G E 1$ inducible-expression strain relative to the wild-type, 130 of them were also $>8$-fold more highly expressed in the $S G E 1$ inducible-expression strain relative to the sgel mutant, while the remaining five genes were, on average, 5.6-fold more highly expressed.

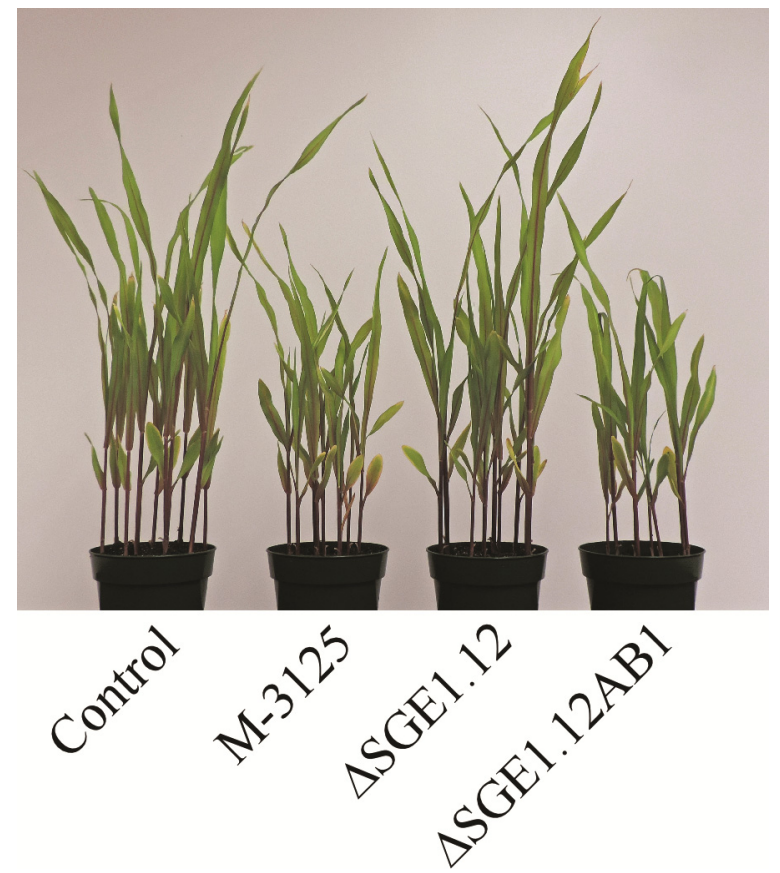

Fig. 3. Results of a seedling blight assay in which conidia of wild-type Fusarium verticillioides (strain M-3125), an sgel mutant (strain $\Delta$ SGE1.12), and a complemented sge1 mutant (strain $\triangle \mathrm{SGE} 1.12 \mathrm{AB} 1$ ) were applied to seeds of 'Silver Queen' maize. Seed were then sown, and seedling height was evaluated 16 days after sowing. The sgel mutant had a small by not significant effect on seedling growth, whereas the wild type and complemented mutant significantly reduced growth.

Table 2. Pathogenicity of complemented sgel mutants of Fusarium verticillioides in a maize seedlings assay

\begin{tabular}{|c|c|c|c|}
\hline Strain $^{a}$ & Genotype & Number of plants ${ }^{b}$ & Mean height $(\mathrm{cm})^{c}$ \\
\hline \multicolumn{4}{|l|}{ Experiment A } \\
\hline None & $\ldots$ & 30 & $36.7 \pm 6.1$ \\
\hline M-3125 & Wild type & 28 & $27.3 \pm 6.1^{\mathrm{d}}$ \\
\hline$\Delta \mathrm{SGE} 1.12$ & sge1 mutant & 26 & $33.1 \pm 7.4$ \\
\hline$\Delta$ SGE1.12AB1 & Complemented sge1 mutant & 26 & $25.9 \pm 7.2^{\mathrm{e}}$ \\
\hline$\Delta \mathrm{SGE} 1.12 \mathrm{AB} 15$ & Complemented sge1 mutan & 30 & $27.1 \pm 8.5^{\mathrm{e}}$ \\
\hline$\Delta$ SGE1.12AB19 & Complemented sge1 mutan & 28 & $29.8 \pm 7.9^{\mathrm{d}}$ \\
\hline \multicolumn{4}{|l|}{ Experiment B } \\
\hline None & $\ldots$ & 30 & $41.1 \pm 9.0$ \\
\hline$\Delta$ SGE1.12 & sgel mutant & 29 & $36.1 \pm 6.6$ \\
\hline$\Delta \mathrm{SGE} 1.12 \mathrm{AB} 1$ & Complemented sge1 mutan & 30 & $37.1 \pm 6.5$ \\
\hline$\Delta \mathrm{SGE} 1.12 \mathrm{AB} 15$ & Complemented sgel mutan & 29 & $29.4 \pm 8.1^{\mathrm{f}}$ \\
\hline$\Delta$ SGE1.12AB19 & Complemented sgel mutan & 30 & $33.4 \pm 7.2^{\mathrm{f}}$ \\
\hline
\end{tabular}

${ }^{a}$ In experiment A, maize seed were inoculated prior to sowing in $10 \mathrm{ml}$ of water with $1 \times 10^{6}$ spores and incubated overnight. Plant heights were assessed after 16 days. In experiment B, corn stalk pieces (approximately 3 by $1 \mathrm{~cm}$ ) were inoculated with a spore suspension and allowed to incubate for 5 days. Five corn stalk pieces were then placed into the soil in each pot $24 \mathrm{~h}$ prior to sowing seed. Plant heights were assessed after 21 days.

${ }^{\mathrm{b}}$ At the time disease was assessed, the number of living maize seedlings above $10 \mathrm{~cm}$ in height that resulted from 30 seeds sown in three pots (10 seeds/pot).

${ }^{\mathrm{c}}$ Height of each seedling was determined by measuring distance from the soil surface to the distal end of the longest leaf.

${ }^{\mathrm{d}}$ Maize seedlings infected with M-3125 had a significantly lower mean height than uninoculated seedlings based on Dunnett's test at $P \leq 0.05$.

${ }^{\mathrm{e}}$ Maize seedlings infected with all three complemented sge1 mutants had significantly lower mean height than seedlings infected with the parent sgel mutant based on Dunnett's test at $P \leq 0.05$.

${ }^{\mathrm{f}}$ Maize seedlings infected with two of the three complemented sgel mutants had a significantly lower mean height than seedlings infected with the parent sge1 mutant based on Student's $t$ test at $P \leq 0.05$. 


\section{Expression of effector genes.}

The $F$. verticillioides genome includes multiple genes that are predicted to encode putative effectors, including predicted cell-wall-degrading enzymes (e.g., glycoside hydrolase [GH]), necrosis-inducing proteins (NEPs), and small, cysteine-rich proteins (de Jonge et al. 2011). Some of these genes are among those affected by $S G E 1$ deletion and induced expression. Of approximately 107 genes predicted to encode small, secreted, cysteine-rich proteins (Ma et al. 2010), 36 exhibited greater than twofold higher expression and only two exhibited greater than twofold lower expression in the $S G E 1$ inducible-expression strain than the sge 1 mutant (Supplementary Table S7). In contrast, 14 exhibited greater than twofold higher expression and none exhibited greater than twofold higher expression in the wild type compared with the sgel mutant. Thus, of the genes encoding small, cysteine-rich proteins that were affected by $S G E 1$, almost all were positively affected.

$S G E 1$ also had a positive effect on expression of GH genes more often than it had a negative effect. Of the 297 putative GH genes in the F. verticillioides genome (Ma et al. 2010), 54 exhibited greater than twofold higher expression and 17 exhibited greater than twofold lower expression in the $S G E 1$ inducible-expression strain compared with the sgel mutant (comparison group 1). Likewise, 30 exhibited greater than twofold higher expression and four exhibited greater than twofold lower expression in the wild type compared with the sgel mutant (comparison group 2) (Supplementary Table S8). As expected, there is considerable overlap between these two groups. The existence of $12 \mathrm{GH}$ genes more highly expressed in the wild type compared with the sgel mutant suggests that

Table 3. Secondary metabolite production in sgel deletion mutants ( $\triangle \mathrm{SGE} 1$ strains), complemented sge1 mutants $(\triangle \mathrm{SGE} 1.12 \mathrm{AB}$ strains), and wild-type (strain M-3125) Fusarium verticillioides

\begin{tabular}{lcc}
\hline & \multicolumn{2}{c}{ Secondary metabolite } \\
\cline { 2 - 3 } Strain & Fumonisins $^{\mathbf{a}}$ & Fusarin $^{\mathbf{b}}$ \\
\hline Experiment A & $350( \pm 136)$ & $250( \pm 27)$ \\
M-3125 $^{\mathrm{c}}$ & $130( \pm 63)$ & $352( \pm 48)$ \\
$\Delta$ SGE1.6 & $146( \pm 47)$ & $348( \pm 18)$ \\
$\Delta$ SGE1.12 & $296( \pm 15)$ & $226( \pm 83)$ \\
GMTcp-272 & & \\
Experiment B & $137( \pm 158)$ & NA \\
M-3125 & $22( \pm 25)$ & NA \\
$\Delta$ SGE1.12 & $94( \pm 18)$ & NA \\
$\Delta$ SGE1.12AB1 & $43( \pm 16)$ & NA \\
$\Delta$ SGE1.12AB15 & $99( \pm 119)$ & NA \\
$\Delta$ SGE1.12AB19 & $105( \pm 21)$ & NA \\
GMTcp-272 &
\end{tabular}

${ }^{a}$ Total fumonisins was determined by combining the amount of $\mathrm{FB}_{1}, \mathrm{FB}_{2}$, and $\mathrm{FB}_{3}$ detected.

${ }^{\mathrm{b}} \mathrm{NA}=$ not available

${ }^{c}$ Levels $(\mathrm{ng} / \mu \mathrm{l})$ were determined in extracts of three cultures per strain.

${ }^{\mathrm{d}}$ Levels $(\mathrm{ng} / \mu \mathrm{l})$ were determined in extracts of two cultures per strain. loss of $S G E 1$ affects some genes more than induced expression of $S G E 1$. Taken together, these results provide further evidence that $S G E 1$ can serve to positively and negatively regulate transcription, even of the same gene, depending on context.

The $F$. verticillioides genome includes three genes (FVEG_ 04647, FVEG_10941, and FVEG_13350) that are predicted to encode NEPs. Expression of FVEG_10941 was greater than fivefold higher in the SGE1 inducible-expression strain compared with the sgel mutant, and greater than twofold higher in the wild type compared with the sgel mutant. In contrast, expression of FVEG_04647 and FVEG_13350 did not differ significantly in the sgel mutant compared with either the $S G E 1$ inducible-expression strain or the wild type.

Orthologs of multiple effector genes exist in both $F$. verticillioides and $F$. graminearum. However, few of these genes that were affected by $S G E 1$ in $F$. verticillioides appear to be affected in the same manner by the SGE1 ortholog, FGP1, in $F$. graminearum (Jonkers et al. 2012). Of the $14 \mathrm{~F}$. verticillioides cysteine-rich protein-encoding genes with lower expression in the sgel mutant relative to the wild type, five have orthologs in $F$. graminearum. In a similar manner, of the $30 \mathrm{~F}$. verticillioides $\mathrm{GH}$ effector genes with significantly reduced expression (greater than twofold reduction) in the sgel mutant relative to the wild type, 20 have orthologs in $F$. graminearum. $F$. graminearum has orthologs of all three putative $F$. verticillioides NEP genes but none were reported to be transcriptionally affected by FGP1 (Jonkers et al. 2012).

\section{Expression of SM biosynthetic genes and gene clusters.}

Because of the reduced production of fumonisins and increased production of fusarins in sgel mutants, we also assessed the effect of deletion and induced expression of SGE1 on expression of genes required for synthesis of these and other SM. For this assessment, we utilized the microarray data from the experiment described above (i.e., SYP medium cultures; addition of xylose at $18 \mathrm{~h}$; and sampling 5, 30, and 360 min later). A potential limitation of the experiment for such an analysis is that, in laboratory culture media, expression of SM biosynthetic genes is typically low at and before $24 \mathrm{~h}$ of overall growth. Nevertheless, differences in expression of some genes were observed. Expression of the fumonisin and fusarin polyketide synthase (PKS) genes (FUMI and FUS1, respectively) was low and essentially unchanged in the sgel mutant relative to the wild type. In contrast, FUM1 expression was 1.7-fold higher and FUS1 expression was 13-fold higher in the $S G E$ inducibleexpression strain compared with the sgel mutant (Table 5).

Differences in expression indicate that five other $F$. verticillioides PKS genes may also be regulated by SGE1. A comparison of the wild type versus the sgel mutant expression levels revealed that one gene, FVEG_00079, was >14-fold more highly expressed in the wild type (Table 5). In contrast, four PKS genes (bikaverin PKS [BIK1], FVEG_00079, FVEG_ 10497, and FVEG_08425) exhibited greater than twofold higher

Table 4. Numbers of genes exhibiting increased or decreased expression as a result of deletion or induced expression of $F v S G E 1^{\mathrm{a}}$

\begin{tabular}{|c|c|c|c|c|c|c|}
\hline \multirow[b]{2}{*}{ Fold change } & \multicolumn{2}{|c|}{$\begin{array}{l}\text { Wild type (SGE1) vs. } \\
\text { sge1 mutant (sge1) }\end{array}$} & \multicolumn{2}{|c|}{$\begin{array}{l}\text { Wild type (SGE1) vs. inducible- } \\
\text { expression strain (IESGE1) }\end{array}$} & \multicolumn{2}{|c|}{$\begin{array}{l}\text { sge } 1 \text { mutant (sge1) vs. inducible- } \\
\text { expression strain (IESGE1) }\end{array}$} \\
\hline & SGE1 > sge1 & sge1 > SGE1 & $S G E 1>$ IESGE1 & IESGE1 > SGE1 & sge1 > IESGE1 & IESGE1 > sge1 \\
\hline $1-2$ & 1,461 & 1,103 & 2,104 & 1,526 & 2,720 & 1,879 \\
\hline $2-4$ & 544 & 108 & 829 & 566 & 505 & 952 \\
\hline $4-8$ & 110 & 25 & 148 & 145 & 93 & 212 \\
\hline$>8$ & 58 & 9 & 53 & 135 & 24 & 223 \\
\hline Total $^{\mathrm{b}}$ & 2,173 & 1,245 & 3,134 & 2,372 & 3,342 & 3,266 \\
\hline
\end{tabular}

${ }^{a}$ Strains used in this experiment were wild-type strain M-3125, sge1 mutant strain $\triangle$ SGE1.12, and SGE1 inducible-expression strain GMTcp-272. Values are numbers of genes exhibiting increase or decrease in expression.

${ }^{\mathrm{b}}$ Fold change in expression. Total $=$ total number of genes that exhibited increased or decreased expression in strain comparisons indicated $(P<0.01)$. 
expression and one PKS gene, FVEG_10535, exhibited twofold lower expression in the $S G E 1$ inducible-expression strain compared with the sgel mutant (Table 5).

The microarray data indicate that the expression of genes flanking some of the PKS genes exhibited changes in expression in the sgel mutant relative to the wild type or SGE1 inducibleexpression strains in a manner similar to changes exhibited by the PKS genes. The coregulation of genes within the FUM, $F U B, F U S$, and $B I K$ gene clusters has been previously described (Brown et al. 2012). The nine genes in the FUS cluster exhibited similar changes in expression in the mutant relative to both the wild type and the $S G E 1$ inducible-expression strain (Fig. 4), whereas genes within other clusters did not always exhibit similar changes in expression. For example, only four of 17 FUM genes and three of six $B I K$ genes were more highly expressed in the $S G E 1$ inducible-expression strain than the sgel mutant. The lack of similar patterns of expression for genes within the FUM and $B I K$ clusters likely reflects low transcript levels for the genes at $18.5 \mathrm{~h}$ of incubation, the timepoint at which the transcriptome was examined here, rather than dissolution of coregulation of the clusters in the sgel mutant or SGE1 inducible-expression strain over longer time frames.

Similar to expression patterns of the FUS cluster, three PKS genes and one or two genes adjacent to them exhibited similar changes in expression in response to $S G E 1$ deletion or induced expression. First, PKS gene FVEG_08425 and the putative dehydrogenase gene, FVEG_08426, adjacent to it both exhibited significantly higher (twofold) levels of expression in the $S G E 1$ inducible-expression strain compared with the sgel mutant. Second, PKS gene FVEG_10535 and the putative methyltransferase gene, FVEG_10536, adjacent to it both exhibited significantly lower levels of expression in the SGE1 inducibleexpression strain compared with the sgel mutant. Finally, PKS gene FVEG_00079 and two genes, FVEG_00078 and FVEG_ 00077, contiguous with it all exhibited much higher (23-fold average) levels of expression in the wild type than the sgel mutant. This three-gene cluster has been described previously (Ma et al. 2010) and is conserved in the genome sequences of $F$. verticillioides, $F$. fujikuroi, F. pseudograminearum, F. graminearum, and some strains of $F$. oxysporum. In $F$. graminearum, two of the three genes in the cluster exhibit greater than twofold higher levels of expression in the wild type than the fgpl (= sgel) mutant (Jonkers et al. 2012). In addition to the $\mathrm{PKS}$, the cluster encodes a putative G-protein coupled receptor (GPCR) of the Pth11 family (FVEG_00078) (Ma et al. 2010) and a putative acyltransferase (FVEG_00077). PTH11 was originally described in Magnaporthe spp. and is required for host surface recognition prior to appressorium development (DeZwaan et al. 1999). The SM products of this cluster have not yet been determined for any species of Fusarium.

Table 5. Effect of $S G E 1$ on expression of Fusarium verticillioides $P K S$ genes ${ }^{\mathrm{a}}$

\begin{tabular}{|c|c|c|c|c|c|}
\hline \multirow[b]{2}{*}{ PKS } & \multirow[b]{2}{*}{ WT } & \multirow[b]{2}{*}{ sge1 } & \multirow[b]{2}{*}{ IESGE1 } & \multicolumn{2}{|c|}{ Fold difference in expression } \\
\hline & & & & WT vs. sge1 & IESGE1 vs. Sge 1 \\
\hline$F U M 1^{\mathrm{b}}$ & 5.4 & 4.9 & 5.7 & nd & 1.7 \\
\hline$F U S 1^{\mathrm{b}}$ & 8.5 & 8.2 & 11.9 & nd & 13.0 \\
\hline$F U B 1^{\text {b }}$ & 6.2 & 6.1 & 6.2 & nd & nd \\
\hline FVEG_00079 & 8.5 & 4.7 & 9.5 & 14.0 & 27.9 \\
\hline BIK1 & 5.9 & 5.3 & 6.8 & nd & 2.8 \\
\hline FVEG_10497 & 4.8 & 4.2 & 5.7 & nd & 2.8 \\
\hline FVEG_08425 & 4.8 & 3.9 & 5.1 & nd & 2.3 \\
\hline FVEG_10535 & 13.8 & 13.5 & 12.5 & nd & -2.0 \\
\hline PGL1 & 6.0 & 6.0 & 6.9 & nd & nd \\
\hline FVEG_12610 & 5.3 & 4.6 & 5.4 & nd & nd \\
\hline FVEG_09961 & 5.2 & 4.5 & 5.4 & nd & nd \\
\hline FVEG_01914 & 9.6 & 9.2 & 9.0 & nd & nd \\
\hline FVEG_11932 & 8.3 & 8.6 & 9.5 & nd & nd \\
\hline FVEG_13715 & 5.7 & 5.0 & 5.6 & nd & nd \\
\hline FVEG_05537 & 7.0 & 7.3 & 6.9 & nd & nd \\
\hline FVEG_01736 & 4.9 & 4.5 & 5.1 & nd & nd \\
\hline
\end{tabular}

${ }^{a}$ Wild type (WT), sge1 deletion mutant (sge1), and $S G E 1$ inducible-expression strain (IESGE1) expression values are indicated in log2 scale. The change in expression for each gene was calculated by determining the differences in $\log 2$ scale value determined for each gene and converting it to a linear value; nd $=$ no significant difference.

${ }^{\mathrm{b}}$ Genes corresponding to secondary metabolites for which production levels were determined.

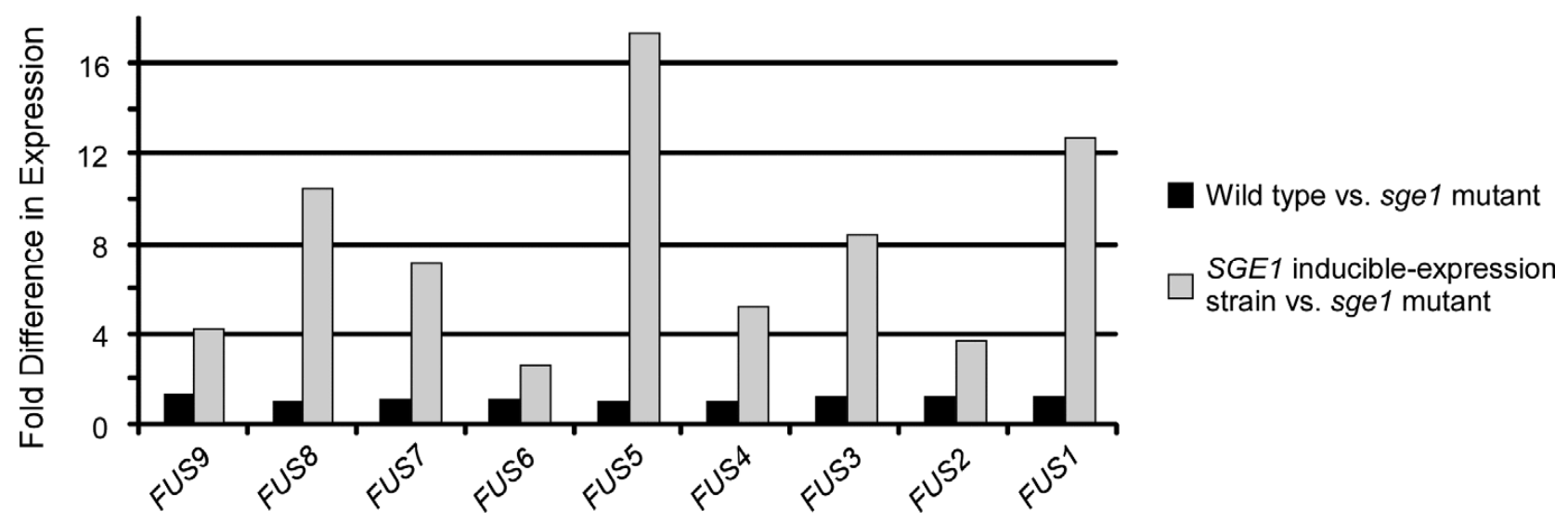

Fig. 4. Differences in expression of nine FUS cluster genes in the wild type (strain M-3125) versus an sge1 mutant (strain $\triangle$ SGE1.12) and in sge1 mutant versus SGE1 inducible-expression strain (GMTcp-272) of Fusarium verticillioides. 

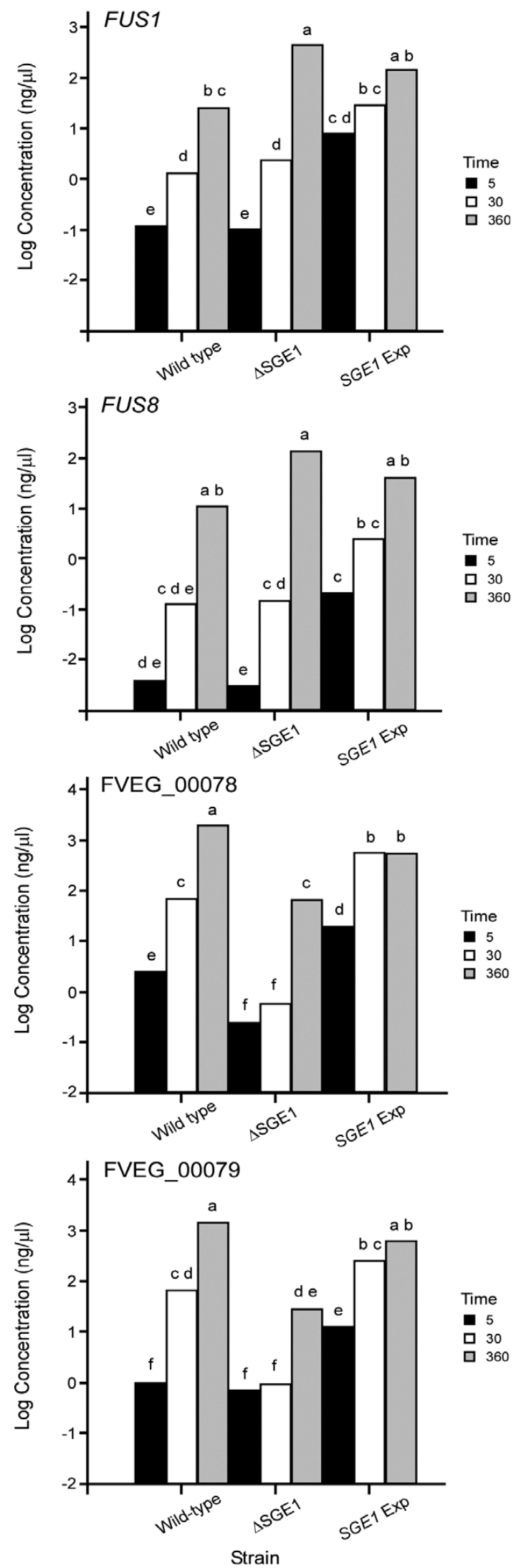

Fig. 5. Results of quantitative polymerase chain reaction (qPCR) analysis of four likely secondary metabolite biosynthetic genes in wild-type strain

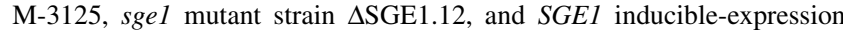
strain GMTcp-272 (SGE1 Exp). After $18 \mathrm{~h}$ of incubation, cultures were induced by the addition of $50 \mathrm{mM}$ xylose, and mycelia were harvested for RNA preparation 5, 30, and 360 min later. The genes analyzed were FUS1, FUS8, FVEG_00078, and FVEG_00079. Columns with the same letters in the same graph are not significantly different.
Validation of gene expression in FUS and FVEG_00079 clusters.

To validate the microarray results, we performed quantitative PCR (qPCR) analysis with four genes that appeared to be regulated by $S G E 1$. These genes were also chosen because they are likely to be involved in secondary metabolism, which is a major focus of this study. FUS1 and FUS8 are part of the FUS gene cluster and encode a putative PKS and monooxygenase, while FVEG_00079 and FVEG_00078 encode a putative PKS and GPCR, respectively. qPCR was carried out in triplicate for three biological replicas for each gene at 5, 30, and $360 \mathrm{~min}$ after induction with xylose. Analysis of variance (ANOVA) of data for each gene showed significant differences $(P<0.05)$ in expression between the strain-time interactions. In agreement with the microarray results, FUS1 expression values were significantly different in the SGE1 inducibleexpression strain compared with the wild type and the sgel mutant at 30 min (Fig. 5A). Although FUS8 expression was not significantly different in the three strains after $30 \mathrm{~min}$, it was significantly different after $5 \mathrm{~min}$ (Fig. 5B). Also in agreement with the microarray results, both FVEG_00078 and FVEG_00079 expression levels were lower in the sge1 mutant at the 30-min timepoint compared with both the $S G E 1$ inducible-expression strain and the wild type (Fig. 5C and D). We also found a significant change in expression for all four genes in the wild type between 5 and 30 min, possibly an effect of the inducing agent xylose. In contrast, no significant change was observed for either FVEG_00078 or FVEG_00079 in the sgel mutant between 5 and $30 \mathrm{~min}$ or for all four genes between 30 and $360 \mathrm{~min}$. This lack of change could reflect interactions or an overlap between the effects of xylose and $S G E 1$ on the transcriptome. Overall, the data indicate that $S G E 1$ can regulate some SM biosynthetic genes, but additional analyses are necessary to further elucidate the mechanism by which this regulation occurs.

\section{Expression of genes associated with conidiation.}

Although SGE1 deletion did not have a noticeable effect on conidiation in $F$. verticillioides, it does affect conidiation in other plant-pathogenic fungi that have been examined (Jonkers et al. 2012; Michielse et al. 2009b, 2011; Santhanam and Thomma 2013). Therefore, we examined the effect of SGE1 deletion and induced expression on the expression of potential conidiation genes in $F$. verticillioides. To do this, we utilized microarray data to assess expression of five genes previously shown to be involved in conidiation in $F$. verticillioides as well as $F$. verticillioides homologs of five genes that affect conidiation in other fungi (Supplementary Table S9). Expression of the $F$. verticillioides homologs of the REN1, ABA1, FLBC, FLBA1, FLBA2, STUA, and HYD2 genes did not differ significantly in the sgel mutant or $S G E 1$ inducible-expression strain compared with the wild type. In contrast, expression of the $F$. verticillioides homolog of NPS2, which is involved in spore cell wall development and hydrophobicity in Alternaria brassicicola and Cochliobolus heterostrophus (Kim et al. 2007), was greater than fourfold lower in the sgel mutant relative to the wild type and 2.5-fold higher in the SGE1 inducible-expression strain relative to the sge1 mutant. Expression of HYD2, which encodes a putative hydrophobin required for conidiation in F. verticillioides (Fuchs et al. 2004), did not differ in the sgel mutant relative to the wild type but was 3.5-fold lower in the $S G E 1$ inducible-expression strain relative to the sgel mutant. Although we did not observe any obvious effect of the loss of $S G E 1$ on conidiation in the sgel mutants, the changes in gene expression for some genes involved in conidiation in other fungi in the sgel mutant and in the SGEl induced-expression strain suggests that additional studies are warranted. 


\section{Expression of membrane proteins.}

Cell surface structures are likely a critical aspect of how fungi transition between phases of their lifecycles because such structures contribute to how fungi present themselves to their environment and how they sense extracellular molecules in the environment. If $S G E 1$ contributes to the ability of fungi to manage this transition, the expression of genes encoding cell surface proteins would likely be effected by the appearance or loss of SGE1. Two large groups of cell surface proteins are GPCRs and secreted anchored (SA) proteins. GPCRs are a large family of transmembrane domain proteins that bind ligands outside the cell and activate signal transduction pathways inside the cell. Of the 102 putative GPCRs identified in $F$. verticillioides (Ma et al. 2010), 29 exhibited greater than twofold lower expression and two exhibited greater than twofold higher expression in the sgel mutant compared the wild type. In contrast, 20 exhibited greater than twofold higher expression and 12 exhibited greater than twofold lower expression in the $S G E 1$ inducible-expression strain compared with the sgel mutant (Supplementary Table S10).

SA proteins contain a putative secretion signal peptide and can be further divided into two groups based on whether they are predicted to be glycophosphatidylinositol (GPI) anchored to a membrane or whether they contain a unique pentapeptide $[\underline{G S}][\underline{P A V N K R]} \underline{\mathrm{C}}[\underline{\mathrm{R} K H N}] \underline{\mathrm{P}}$ (referred to as GPCRP proteins) after the signal peptide (Ma et al. 2010). Of the 128 predicted SA genes, six exhibited greater than twofold lower expression and 25 exhibited greater than twofold higher expression in the sgel mutant compared with the wild type. In contrast, 18 exhibited greater than twofold higher expression and 26 exhibited greater than twofold lower expression in the SGE1 inducibleexpression strain compared with the sgel mutant (Supplementary Table S11). Seventeen of the SA genes appear to be negatively regulated by $S G E 1$ in that they exhibited significantly (greater than twofold) higher expression in the sgel mutant than in the wild type and significantly lower expression in the $S G E 1$ inducible-expression strain than in the wild type. The predicted SA proteins encoded by these 17 genes include the GPCRP peptide.

Included in the SA-encoding genes negatively regulated by SGE1 are six that share significant similarity to one another (Supplementary Table S12). Two other genes encode secreted proteins that are not anchored and also share significant similarity to each other and the six GPCRP proteins. Transcripts for this family of eight genes in the sgel mutant range from 4.1-fold to $>23$-fold more than the wild type (Supplementary Table S13). Expression of all eight genes was significantly less in the wild type compared with the sgel mutant (mean 8.8-fold difference) but significantly greater in the SGE1 inducibleexpression strain compared with the sgel mutant (mean 5.5fold difference). To what extent these genes are regulated by $S G E 1$ and whether and how they contribute to the ability of $F$. verticillioides to live as a saprophyte, endophyte, or pathogen remain to be determined.

\section{Differing effects of $S G E 1$ among Fusarium spp.}

Previously, Jonkers and associates (2012) carried out a microarray-based transcriptomic analysis of an fgpl (= sgel) mutant and wild-type strain of $F$. graminearum. Here, we compared these $F$. graminearum data with data generated in the current study for equivalent strains of $F$. verticillioides in order to compare the types of genes regulated by $S G E 1$ orthologs in different species of Fusarium. The data sets are not exactly equivalent because the cultures used to prepare RNA for each species employed different growth media and incubation times. Nevertheless, comparisons of the two data sets were informative. Approximately $64 \%$ of the genes in the F. verticil- lioides and $F$. graminearum genomes are orthologous (Ma et al. 2010). Of the $774 F$. verticillioides genes that exhibited greater than twofold lower expression in the $F$. verticillioides sgel mutant versus the wild type, 372 have orthologs in $F$. graminearum. Of these 372 orthologs, 57 exhibited greater than twofold lower expression and 33 exhibited greater than twofold higher expression in the $F$. graminearum fgpl mutant compared with its wild-type progenitor. Of the $156 \mathrm{~F}$. verticillioides genes with greater than twofold higher expression in the $F$. verticillioides sge1 mutant versus the wild-type, 100 had orthologs in F. graminearum. Of these 100 orthologs, six exhibited greater than twofold higher expression and six others exhibited greater than twofold lower expression in the fgpl mutant compared with the wild type. In a similar manner, we compared our data with preliminary transcriptome data for the wild type and an sgel mutant of $F$. fujikuroi grown under a third set of growth conditions (C. Michielse and B. Tudzynski, personal communication). Approximately $71 \%$ of the genes in the $F$. verticillioides and $F$. fujikuroi are orthologous (Wiemann et al. 2013). Of the $774 F$. verticillioides genes with greater than twofold lower expression in the sgel mutant versus the wild type, 421 have orthologs in F. fujikuroi. Of these 421 genes, 36 exhibited greater than twofold lower expression and 21 exhibited greater than twofold higher expression in $F$. fujikuroi sgel mutant compared with its wild-type progenitor. Of the $156 \mathrm{~F}$. verticillioides genes with greater than twofold higher expression in the sgel mutant versus the wild type, 85 have orthologs in F. fujikuroi. Five of these orthologs exhibited greater than twofold lower expression and six exhibited greater than twofold higher expression in the F. fujikuroi sgel mutant compared with the wild type. These comparisons indicate that SGE1 regulates markedly different sets of genes in different species of Fusarium, a finding that is consistent with a comparative analysis of sgel/fgpl mutants of $F$. graminearum and F. oxysporum (Jonkers et al. 2012).

Despite this general observation, we identified seven orthologs with greater than twofold lower expression in sgel mutants of $F$. verticillioides, $F$. fujikuroi, and $F$. graminearum. Two of the seven genes are adjacent to each other in all three organisms; one of these, FVEG_07524, is predicted to encode a sugar transporter and the other, FVEG_07525, a glycoside hydrolase. Three of the seven genes are predicted to encode secreted proteins: a lipase (FVEG_09233), a chitinase (FVEG_08470), and a pectinesterase (FVEG_10795). The final two genes are predicted to encode an amino acid lyase (FVEG_10108) and a protein involved in synthesis of amino-sugars (FVEG_02064). Although the similar regulation of these genes by SGE1 implies a similar function, whether it be virulence, secondary metabolism, or other phenotype in the three Fusarium spp., how they contribute in $F$. verticillioides remains to be determined.

\section{$S G E 1$ regulated genes that are not required for disease on maize.}

We used gene deletion analysis to assess the role in pathogenicity of seven other $F$. verticillioides genes that are positively regulated by $S G E 1$. Also, some of these genes are homologs of genes that have been shown to play a role in disease in other fungi. For example, FVEG_02173 has a homolog (FOXG_03305) in F. oxysporum f. sp. lycopersici that contributes to pathogenicity on tomato (Michielse et al. 2009a). FVEG_02431 has a homolog in Metarhizium anisopliae that is linked to virulence on insects (Wang and St Leger 2007). However, F. verticillioides mutants in which either FVEG_02173 or FVEG_02431 were deleted exhibited wild-type levels of virulence in the maize seedling assay (Supplementary Table S14).

Killer protein 4 (KP4) is a small, cysteine-rich viral toxin that inhibits Fusarium and plant root growth and is secreted by 
the maize smut fungus Ustilago maydis (Allen et al. 2008; Gage et al. 2002). Previously, we identified six KP4-like (killer protein-like $[K P L]$ ) genes in the genome of $F$. verticillioides. Five of the six $K P L$ genes encode small, secreted, cysteine-rich proteins and, thus, could serve as effectors (Brown 2011). Microarray analysis of the five putative effectors revealed that expression of $K P L 1, K P L 3$, and $K P L 5$ was $2.4-$ to 5.1-fold (average 3.7-fold) lower in the sgel mutant compared with the wild type. Only KPL5 was greater than twofold more highly expressed in the $S G E 1$ inducible-expression strain compared with the sgel mutant. $F$. verticillioides mutants lacking $K P L 1, K P L 2$, and KPL3 (a triple gene mutant) or strains lacking either KPL5 or KPL6 caused levels of disease similar to the wild type. Again, despite being regulated by $S G E 1, K P L$ genes did not contribute significantly to pathogenicity of $F$. verticillioides on maize seedlings under the conditions examined.

\section{DISCUSSION}

Fungi can live and compete in widely diverse habits, including as saprophytes on virtually any carbon source or in commensal, mutualistic, or parasitic relationships with plants, animals, insects, or other fungi. How plant-associated fungi interact with their host can change with time and environment. For example, F. verticillioides can exist as a saprophyte, endophyte, and parasite over a single growing season. The ability of fungi to alter the physiology of plant hosts in order to live endophytically or parasitically has been attributed to production as well as nonproduction of effectors, including small, cysteine-rich proteins, cell-wall-degrading enzymes, necrosisinducing proteins, and SM (de Jonge et al. 2011; Rafiqi et al. 2012). Because of its importance in pathogenicity and its ability to regulate effector-encoding genes in other fusaria, we characterized $F$. verticillioides SGE1 to begin to elucidate genetic regulation of effectors and their relationship to endophytism and parasitism in this maize-associated fungus. We found that SGE1 did not affect saprophytic growth on maize stalks and a variety of media, whereas pathogenicity on seedlings was affected. One possible explanation is that $S G E 1$ plays a role in the transition of the fungus from a saprophyte on maize stalks to growth within the plant as an endophyte or parasite. Although Sge1 orthologs in other plant-pathogenic fungi have been found to be important for disease, this is the first characterization of a role in lifestyle transition, similar to what was first noted in yeast.

Included in our approaches to examine the role of $S G E 1$ in regulation of gene expression was the generation of the $S G E 1$ inducible-expression strain of $F$. verticillioides, in which the $S G E 1$ coding region was fused to the promoter region of a gene encoding a putative xylanase $(X L N 2)$, such that expression of $S G E 1$ could be induced by addition of xylose to culture media. This approach to controlled gene expression has been utilized successfully in Penicillium and Aspergillus spp. (Wong et al. 2009; Zadra et al. 2000). The major advantage is that it can reveal genes first induced by the rapid appearance of Sge1: those more likely directly regulated. Our results demonstrate that this inducible-expression system resulted in rapid and high levels of expression of $S G E 1$, within 5 min of induction, and can lead to expression of $S G E 1$-regulated genes within $30 \mathrm{~min}$ (Fig. 2). A limitation of this system is that xylose induced transcriptional changes over time for three of the four genes we examined by qPCR (Fig. 5). In our microarray experiment, this limitation was controlled for by comparing transcriptional differences between similarly treated strains at a single timepoint. Our results indicate that the $X L N 2$ promoter system could be a useful tool for characterization of other regulatory or structural genes in $F$. verticillioides.
The results reported here indicate that the $F$. verticillioides SGE1 affects a lifestyle transition (saprophyte to pathogen), secondary metabolism, and the expression of hundreds of genes but does not affect vegetative growth or conidiation under the laboratory conditions examined. Our results also indicate that the effect of $S G E 1$ on pathogenicity in the genus Fusarium differs among species. In $F$. graminearum and $F$. oxysporum, SGE1 deletion almost completely abolished pathogenicity in wheat head blight and tomato seedling wilt assays, respectively. In F. graminearum, pathogenicity and SM synthesis are connected; trichothecene production is required for spread of the fungus between wheat spikelets (Bai et al. 2002; Seong et al. 2009). F. graminearum fgpl deletion mutants are unable to synthesize trichothecenes and cause only minimal disease in the inoculated spikelet (Jonkers et al. 2012). This effect on virulence may be related to general growth developmental defects in the mutants or to a direct role of $S G E 1$ in the initial infection. In contrast, $S G E 1$ deletion in $F$. verticillioides resulted in reduced virulence compared with the wild type but it did not abolish pathogenicity. In addition, $S G E 1$ deletion did not completely block production of any of the $F$. verticillioides SM examined; fumonisin production was reduced, fusaric acid production was variable, and fusarin production was enhanced in sgel mutants. Although no clear role for an F. verticillioides $\mathrm{SM}$ in pathogenicity has yet been established, the impact of SGE1 on expression of some, unknown PKSs (e.g., FVEG_ 00079) but not others over the time frame of the experiment are intriguing and will be examined further.

The mechanism by which $S G E 1$ reduces fumonisin and increases fusarin production is not clear. Transcriptomic analysis over a longer time frame may help to determine whether the respective changes in production are due to changes in FUM and FUS gene expression. Expression of most genes shown previously to affect fumonisin production ( FCC1, FCK1, LAE1, VE1, AREA, PAC1 HXK1, ZFR1, and FST1) (Woloshuk and Shim 2013) were not significantly different in all three strains. The only gene previously shown to impact fumonisin synthesis found to differ significantly was FUM21, the fumonisin pathway-specific regulatory gene, which exhibited twofold lower expression in the sgel mutant compared with the wild type and 3.6-fold higher expression in the $S G E 1$ inducible-expression strain compared with the sgel mutant. A twofold reduction in FUM21 expression could lead to the observed $50 \%$ reduction in fumonisin production over 10 days. Alternatively, carbon or nitrogen depletion or changes in culture $\mathrm{pH}$ could have affected fumonisin synthesis over the incubation period without an early impact on $A R E A, P A C 1$, or any of the other genes mentioned above.

In contrast to the FUM cluster, the response of the entire FUS cluster to $S G E 1$ (Fig. 4) after 30 min suggests that either Sge1 directly affects transcription by binding to FUS gene promoters or it induces transcription of another gene that then activates expression. The FUS gene cluster is unusual among SM gene clusters in that it does not include a transcription factor gene (Niehaus et al. 2013). An ability of Sge1 to bind FUS gene promoter sequence could be examined by promoter binding or gel shift assays (Fernandes et al. 1998). The SGE1 inducible-expression strain could be used to help identify a possible intermediate transcriptional regulator by accessing the transcriptome at earlier timepoints after induction.

Membrane proteins play a host of critical functions ranging from allowing cells to communicate with their environment to controlling a variety of important metabolic processes. The effect of SGE1 on the transcription of numerous GPCRs and SA proteins suggests a role for these two major groups of membrane proteins in pathogenicity or lifestyle change. A decrease in GPCRs can occur after stimulation and is one mecha- 
nism to control their responsiveness (Claing et al. 2002). The appearance of $S G E 1$ transcripts potentially bypassed the role of one or more of the GPCRs apparently negatively regulated by $S G E 1$, precipitating their downregulation. We are currently examining the roles that some of these GPCRs play in pathogenicity by gene deletion. In contrast, the unknown function of the vast majority (48 of 53) of SA genes precludes much speculation as to their role in virulence or lifestyle change. These effects on GPCR and SA gene expression indicate that SGE1 likely impacts the content of GPCR and SA proteins in the cell membrane of $F$. verticillioides and, thereby, contributes to the ability of the fungus to sense and adapt to environmental changes.

In summary, similar to the $S G E 1$ orthologs described in other plant-pathogenic fungi, $F$. verticillioides $S G E 1$ is required for wild-type levels of virulence and affects secondary metabolism. A comparison of the transcriptomes of the wild type, the sgel mutant, and the SGE1 inducible-expression strain provided significant insight into the genes regulated by $S G E 1$. The number of different genes upregulated by $S G E 1$ in the inducible-expression strain compared with those downregulated in the sgel deletion mutant was significant. The observation by Jonkers and associates (2012) and in the current study that few orthologous genes are regulated by $S G E 1$ in the three Fusarium spp. thus far examined may reflect the predominant use of sgel deletion mutants in these studies and when the transcriptome was sampled. Perhaps more orthologous genes could be found by comparing genes induced in different Fusarium spp. following artificial induction of $S G E 1$, as was done in the current study. Although previous experiments indicated that fusarin is not required for disease, the rapid expression of the entire FUS gene cluster after the induction of $S G E 1$ suggests that it may play a role in fungusplant interactions. Experiments are underway to better understand the role of this metabolite as well as to determine the chemical product resulting from the PKS encoded by FVEG_ 00079 and coregulated genes and determine whether they are required for disease or other aspects of the maize-F. verticillioides interaction.

\section{MATERIALS AND METHODS}

\section{Strains and media.}

The wild-type $F$. verticillioides strain used in this study was M-3125 (NRRL 13447) (Leslie et al. 1992). Strains $\triangle$ SGE1.6, $\triangle$ SGE1.12, and $\triangle$ SGE1.28 were derived from M3125 by deletion of the $S G E 1$ gene. The $S G E 1$ inducibleexpression strain GMTcp-272 and the complemented sgel mutants $\triangle$ SGE1.12AB1, $\Delta$ SGE1.12AB15, and $\triangle$ SGE1.12AB19 were derived from strain $\triangle \mathrm{SGE} 1.12$. All strains were routinely maintained on V8 juice agar (Tuite 1969). Conidial production was assayed by growth of strains in liquid CMC medium (15 g of carboxymethylcellulose [Sigma-Aldrich, St. Louis], $1 \mathrm{~g}$ of $\mathrm{NH}_{4} \mathrm{NO}_{3}, 1 \mathrm{~g}$ of $\mathrm{KH}_{2} \mathrm{PO}_{4}, 0.5 \mathrm{~g}$ of $\mathrm{MgSO}_{4}$. $7 \mathrm{H}_{2} \mathrm{O}$, and $1 \mathrm{~g}$ of yeast extract [Becton, Dickenson and Company, Franklin Lakes NJ, U.S.A.] per liter), GYP medium ( $2 \%$ glucose, $1 \%$ peptone, and $0.3 \%$ yeast extract), solid GYP (GYP plus 1.5\% agar), and V8 juice agar plates. RNA was prepared from cultures grown in SYP medium $(100 \mathrm{mM}$ sorbitol, $0.25 \%$ peptone, and $0.075 \%$ yeast extract) at $28^{\circ} \mathrm{C}$ and $200 \mathrm{rpm}$. For fumonisin, fusarin $\mathrm{C}$, and fusaric acid production, strains were grown in cracked maize kernel medium for 10 days at $25^{\circ} \mathrm{C}$ in the dark. The medium was prepared by autoclaving $2.5 \mathrm{~g}$ of cracked maize kernels and $1.2 \mathrm{ml}$ of distilled water in a 4-dram glass vial. Each vial was inoculated with a mycelial plug taken from a V8 juice agar culture of $F$. verticillioides.

\section{Nucleic acid sequence analysis.}

Nucleotide sequences of $F$. oxysporum gene SGE1 (gene model FOXG_10510) and the $F$. verticillioides genes $S G E 1$ (FVEG_09150) and SIX2 (FVEG_03307) were retrieved from the Broad Institute's Fusarium Comparative Database. The nucleotide sequence of the F. oxysporum gene SIX2 (FOXG_ 16416) was retrieved from the National Center for Biotechnology Information (NCBI), accession CAE55868. EST sequences corresponding to FVEG_09150 and FVEG_03307 were retrieved from the $F$. verticillioides Gene Index at the DanaFarber Cancer Institute-Harvard School of Public Health. Preparation of the $F$. verticillioides EST libraries has been described previously (Brown et al. 2007). Nucleotide sequence data were assembled using Sequencher (version 5.0; Gene Codes Corp., Ann Arbor, MI, U.S.A.). Alignments of amino acid and nucleotides were carried out using the DNAMAN software (Lynnon BioSoft, Vaudreuil, Quebec, Canada). Sequence similarity analyses were also done by BLASTX and BLASTP searches against the NCBI nonredundant databases (Altschul et al. 1997; Zhang et al. 1998).

F. verticillioides genomic DNA used in PCR analyses was prepared as previously described (Butchko et al. 2003) but with modifications. Mycelia were scraped from 1-week-old V8 juice agar cultures, placed in $250 \mu \mathrm{l}$ of DNA extraction buffer (200 mM Tris [pH 8.0], $250 \mathrm{mM} \mathrm{NaCl}, 25 \mathrm{mM}$ EDTA, and $0.5 \%$ sodium dodecyl sulfate), and incubated for $10 \mathrm{~min}$ at $65^{\circ} \mathrm{C}$. Organic material was separated by the addition of 250 $\mu \mathrm{l}$ of phenol/chloroform/isoamyl alcohol (25:24:1) to the mixture, which was then vortexed for $30 \mathrm{~s}$ and centrifuged at $15,000 \mathrm{rpm}$ for $5 \mathrm{~min}$. The aqueous phase was transferred to a new tube, and genomic DNA was isolated using the UltraClean DNA Purification Kit (Mo Bio, Solana Beach, CA, U.S.A.) following the manufacturer's directions. The resulting genomic DNA (50 pg) was used in PCR with $5 \mu$ l of Promega GoTaq master mix and $10 \mu \mathrm{M}$ of each primer in a total reaction volume of $10 \mu \mathrm{l}$. PCR was carried out in a PTC-100 PCR thermal cycler (MJ Research, Waltham, MA, U.S.A.) using the following program: $95^{\circ} \mathrm{C}$ for $30 \mathrm{~s}$ followed by $57^{\circ} \mathrm{C}$ for $30 \mathrm{~s}$ followed by $72^{\circ} \mathrm{C}$ for $90 \mathrm{~s}$ for 30 cycles.

Southern analysis was used to characterize the deletion strains. Genomic DNA was digested with AseI, separated by agarose gel electrophoresis, and blotted to nylon membrane. The hybridization probe for this analysis was generated by PCR from genomic DNA with primer pair 1383 and 1384, and corresponded to a 718-bp region upstream of the $S G E 1$ coding region. The amplification product was labeled with ${ }^{32} \mathrm{P}$ utilizing Ready-To-Go DNA labeling beads (GE Healthcare, Buckinghamshire, U.K.). Membranes were hybridized using ULTRAhyb solution (Life Technologies, Grand Island, NY, U.S.A.) and washed following the manufacturer's directions.

\section{Gene deletion of $S G E 1$.}

The SGE1 (FVEG_09150) coding region was deleted via the split-marker approach (Catlett et al. 2002; Turgeon et al. 1987) and protoplast transformation method with the selectable marker gene $H y g B$, which confers resistance to the antibiotic hygromycin B (Catlett et al. 2002; Turgeon et al. 1987). Primers used for amplification of the split-maker construct are listed in Supplementary Table S15. To begin preparation of the splitmarker constructs, four DNA fragments were amplified by PCR. The first fragment ( $5^{\prime}$-Flank) corresponded to the $764 \mathrm{bp}$ immediately upstream of the predicted $S G E 1$ start codon and was amplified with primer pair 1382/1384 from genomic DNA of the wild-type strain of $F$. verticillioides. The second fragment ( $3^{\prime}$-Flank) corresponded to the $779 \mathrm{bp}$ immediately downstream of the predicted SGE1 stop codon and was amplified with primer pair 1385/1387 from wild-type genomic DNA. 
The third fragment $(\mathrm{HY})$ consisted of the $5^{\prime}$ two-thirds of the $H y g B$ gene and was amplified with primer pair M13RL/HY from plasmid pHygAsc (Brown et al. 2002). The fourth fragment (YG) corresponded to the 3' two-thirds of $H y g B$ and was amplified with primer pair M13FL/YG from pHygAsc. Two split-marker constructs were then generated by fusion of fragments $5^{\prime}$-Flank and HY and fusion of fragments $3^{\prime}$-Flank and YG (Catlett et al. 2002; Turgeon et al. 1987). Fusion of 5'Flank and HY employed a final nested PCR step with primers 1383 and HYN, while fusion of $3^{\prime}$-Flank and YG employed a final nested PCR step with primers 1386 and YGN.

To delete the $S G E 1$ coding region, approximately $2 \mu \mathrm{g}$ of both split-marker constructs was introduced into the wild-type $F$. verticillioides strain via protoplast-mediated transformation. Deletion was predicted to result from homologous recombination between DNA sequences of the SGE1 $5^{\prime}$ and $3^{\prime}$ flanking regions in $F$. verticillioides genome and in the split-marker constructs and between the two partial copies of $H y g B$ in the split-marker constructs (Fig. 1). These three homologous recombination events were predicted to result in replacement of the 1,242-bp SGE1 coding region with the 2,500-bp HygB resistance cassette. Following the transformation protocol, genomic DNA of hygromycin-resistant transformants, isolated after growth on V8 agar containing hygromycin B (Sigma) at $300 \mu \mathrm{g} / \mathrm{ml}$, was subjected to three PCR screens to determine whether the $S G E 1$ coding region had been deleted and replaced by $H y g B$. In the first screen, primers 1382 and Hygout2 were predicted to amplify an 800-bp fragment if the $S G E 1$ coding region had been deleted but no fragment if $S G E 1$ was intact (Fig. 1A). In the second screen, primers 1385 and Hygout1 were predicted to amplify an 800-bp fragment if the SGE1 coding region had been deleted and no fragment if the gene was intact (Fig. 1A). In the third screen, primers 1388 and 1389 were predicted to yield a $2.5-\mathrm{kb}$ amplicon if $S G E 1$ was deleted and replaced by $H y g B$ and a $1.2-\mathrm{kb}$ amplicon if $S G E 1$ was intact (Fig. 1B). All transformants that yielded amplicons consistent with $S G E 1$ deletion were reisolated from a single conidium and then reanalyzed by PCR with all three primer pairs.

\section{Complementation of the sge1 mutant.}

The SGE1 complementation plasmid pSGE1C was constructed by first cloning a 2,446-bp PCR fragment that spanned from $1,101 \mathrm{bp}$ upstream of the predicted start codon of $F$. verticillioides $S G E 1$ to 359 bp downstream of the predicted SGE1 stop codon. The fragment was amplified from genomic DNA of the wild-type strain with primers 2145 and 2146 , then cloned into the pT7-Blue-3 cloning vector (pT7Blue perfectly blunt cloning kit, EMD Chemicals, Darmstadt, Germany). A fragment containing the geneticin resistance gene GenR was then cloned into the NotI site, creating the complementation plasmid pSGE1C (Bluhm et al. 2008). pSGE1C was introduced into the genome of sgel mutant strain $\Delta \mathrm{SGE} 1.12$ via protoplast-mediated transformation. Transformants were identified by their ability to grow in the presence of geneticin (Gibco) at $300 \mu \mathrm{g} / \mathrm{ml}$, and by PCR analysis to determine whether they contained SGE1 with primers 2145 and 2146. Transformants that yielded the expected amplicon were reisolated from a single conidium and the resulting isolates were reanalyzed by PCR to confirm the presence of pSGE1C.

\section{Generation of $S G E 1$ inducible-expression strain.}

Generation of a xylose-inducible $S G E 1$ expression vector (pXLN2P::SGE1) employed a previously constructed plasmid, pXLN2P (D. W. Brown, unpublished), that includes $900 \mathrm{bp}$ of DNA corresponding to the region immediately upstream of the F. verticillioides xylanase gene XLN2 (FVEG_12502) coding region (i.e., the $X L N 2$ promoter). pXLN2P also includes $A s c \mathrm{I}$ sites, such that coding regions of genes can be cloned into it immediately downstream of the XLN2 promoter via AscI digestion and ligation. A 1,276-bp fragment corresponding to the SGE1 coding region and terminator sequence was amplified from wild-type $F$. verticillioides genomic DNA with primers 1496 and 1497 and cloned into the pT7-Blue-3 cloning vector. Both primer sequences included an $A s c$ I site that facilitated cloning of the DNA fragment downstream of the XLN2 promoter in pXLN2P (Fig. 2). The resulting plasmid, pXLN2P:: SGE1, was introduced into the genome of strain $\triangle \mathrm{SGE} 1.12$ via protoplast-mediated transformation. To do this, plasmid pXLN2P::SGE1 was co-transformed into protoplasts along with plasmid pKS-Gen, which carries the geneticin resistance gene GenR, so that transformants could be selected by their ability to grow in the presence of geneticin (Gibco) at 300 $\mu \mathrm{g} / \mathrm{ml}$.

Geneticin-resistant transformants were subjected to two PCR screens to determine whether they carried the GenR gene and the chimeric XLN2 promoter::SGE1 (SGE1 inducibleexpression) construct pXLN2P::SGE1. The first screen employed primers 649rb and 650rb, which amplify a 634-bp fragment from GenR. The second PCR screen employed primers 1531 and 574rb, which amplify a 1,291-bp amplicon that spans the XLN2 promoter and SGE1 elements of pXLN2P:: SGE1 (Fig. 2). Transformants that yielded the expected amplicons in both PCR screens were reisolated from a single conidium, and the resulting isolates were reanalyzed by PCR to confirm the presence of GenR and the inducible-expression construct.

\section{Deletion of $S G E 1$ regulated genes.}

Mutation of seven $F$. verticillioides genes positively regulated by $S G E 1$ was accomplished by deletion of their predicted open reading frames via the split-marker, as described above for SGE1. For FVEG_02173, FVEG_02431, KPL5, and KPL6, the deletion amplicons corresponded to an approximately 800bp region upstream of the respective start codon and an approximately 800-bp region downstream of the respective stop codon. The simultaneous deletion of KPL1, KPL2, and $K P L 3$, corresponding to $3.2 \mathrm{~kb}$ of genomic sequence, was accomplished in a similar manner. In the latter case, the first amplicon corresponded to an approximately 800-bp region downstream from the KPL1 stop codon, while the second amplicon corresponded to an approximately 800-bp region downstream from the KPL3 stop codon. Hygromycin-resistant transformants, isolated after growth on V8 agar containing hygromycin B (Sigma-Aldrich) at $300 \mu \mathrm{g} / \mathrm{ml}$, were subjected to three PCR screens to determine whether the targeted genes' coding region had been deleted and replaced by $H y g B$, as described above.

\section{Maize seedling assays.}

Seedling assays were carried out essentially as described previously (Glenn et al. 2008; Larson et al. 2011) with the hybrid white corn Silver Queen. Fungicide-free seed were obtained from Johnny's Selected Seeds, Winslow, ME, U.S.A. Seed were surface sterilized by soaking in $100 \%$ commercial bleach (6.25\% sodium hypochlorite) for $10 \mathrm{~min}$, rinsed with sterile water four times, allowed to soak for $4 \mathrm{~h}$ in sterile water, and then heat shocked at $60^{\circ} \mathrm{C}$ for 5 min. Thirty seeds were then incubated at $27^{\circ} \mathrm{C}$ overnight in $10 \mathrm{ml}$ of water containing $F$. verticillioides at $1 \times 10^{6}$ conidia/ml, with or without $50 \mathrm{mM}$ D-xylose (Sigma). Ten seeds were then sown in autoclaved growing mix (Sunshine Redi-earth, Bellevue, WA, U.S.A.) contained in $10-\mathrm{cm}$ plastic pots. Pots were placed in a growth chamber with the following conditions: $14 \mathrm{~h}$ of light at $30^{\circ} \mathrm{C}$ 
and $10 \mathrm{~h}$ of darkness at $20^{\circ} \mathrm{C}$. Disease symptoms were assessed after 16 days by counting the number of plants and measuring height of each plant from soil to distal end of longest leaf. The inoculum for the modified maize seedling assay was prepared by first cutting dried maize stalks lengthwise and then into approximately $3-\mathrm{cm}$ pieces. The stalks were autoclaved twice for an hour each time prior to inoculation. The stalks were incubated at $28^{\circ} \mathrm{C}$ for 6 days to allow ample growth. Five stalk pieces were placed into the top approximately $6 \mathrm{~cm}$ of autoclaved growing mix per pot. After $24 \mathrm{~h}, 10$ washed seeds were sown per pot around the stalks.

\section{Expression analysis.}

For gene expression analyses, strains of $F$. verticillioides were grown in $25 \mathrm{ml}$ of SYP in $125-\mathrm{ml}$ flasks at $28^{\circ} \mathrm{C}$ and 200 rpm. Cultures were initiated by adding conidia to the SYP medium at final concentration of $1 \times 10^{6} \mathrm{conidia} / \mathrm{ml}$. The inducer, $50 \mathrm{mM}$ D-xylose, was added $18 \mathrm{~h}$ after culture initiation. Mycelia were harvested by vacuum filtration and flash frozen in liquid nitrogen. RNA samples for RT-PCR analysis were prepared from cultures harvested 5, 15, and $30 \mathrm{~min}$ after addition of D-xylose, while RNA samples for microarray and qPCR analysis were prepared from cultures harvested 5, 30, and 360 min after addition of D-xylose. Total RNA was extracted from mycelia using the RNeasy plant mini kit (Qiagen Inc., Valencia, CA, U.S.A.). RNA quality was checked using the 2100 Agilent Bioanalyzer (Agilent Technologies Inc., Santa Clara, CA, U.S.A.) and quantified using the Qubit 2.0 Fluorometer (Thermo Fisher Scientific, Waltham, MA, U.S.A.). Prior to RT-PCR, DNA was removed from 25- $\mu$ l samples of RNA by digestion with DNAse (Thermo Fisher Scientific Turbo DNAFree kit). Approximately $80 \mathrm{ng}$ of RNA served as a template per PCR reaction using the Agilent Easy-A 1-Tube RT-PCR kit (Agilent Technologies, Santa Clara, CA, U.S.A.).

Microarrays were designed and produced by Roche NimbleGen (Madison, WI, U.S.A.) based on 14,383 F. verticillioides gene models obtained from the Fusarium Comparative Database and unique EST from the $F$. verticillioides Gene Index. Each sequence was represented on the array by up to six unique 60mer probes. The probe set was represented twice on each array at randomly assigned positions to provide technical replication. Microarray hybridization, data acquisition, and initial analyses were conducted by Roche NimbleGen, Reykjavik, Iceland. Data were normalized by robust multiarray average (Bolstad et al. 2003; Irizarry et al. 2003) and were compared using Acuity 4.0 microarray analysis software (Molecular Devices Corp, Sunnyvale, CA, U.S.A.). The $S G E 1$ experiment (GEO accession number GSE56664) was conducted with RNA extracted from three biological replicas for the wild-type strain M-3125, the sgel mutant strain $\triangle \mathrm{SGE} 1.12$, and $S G E 1$ induced-expression strain GMTcp-272 30 min after the addition of xylose.

qPCR was carried out in triplicate for three biological replicas for each gene. Single-stranded DNA was generated from RNA isolated from mycelia harvested after 5, 30, and 360 min after the addition of xylose to the medium using the Quantitect RT kit (Qiagen Inc.). PCR reactions were carried out using the Rotor-Gene PCR machine (Qiagen Inc.) utilizing the RotorGene SYBR Green kit (Qiagen Inc.) in $25 \mu \mathrm{l}$. Reaction conditions followed the manufacturer's recommendations: $95^{\circ} \mathrm{C}$ for 5 min to activate the HotStarTaqDNA polymerase followed by 40 cycles of $95^{\circ} \mathrm{C}$ for $5 \mathrm{~s}$ and $60^{\circ} \mathrm{C}$ for $10 \mathrm{~s}$. A melting curve from 50 to $95^{\circ} \mathrm{C}$ with reads every $1^{\circ} \mathrm{C}$ was generated to ensure that individual products were produced. Concentration of DNA in nanograms per microliter were determined by comparison to a standard curve generated for the $\beta$-tubulin gene (FVEG_04081) using primers 490rp and 491rp. The means of three subsamples per flask were used so that three flasks per strain-time interaction were treated as the replication. A completely randomized design mixed-model, two-factor ANOVA was performed comparing calculated concentration (nanograms per microliter) between three strains (the wild type, the sgel mutant, and the SGE1 inducible-expression strain) at 5, 30, and $360 \mathrm{~min}$ separately for four genes (FUS1, FUS8, FVEG_00078, and FVEG_00079). Levene's homogeneity of variance tests were performed to determine whether data transformation was necessary prior to ANOVA. All analyses were performed on transformed data where necessary. Treatment comparisons were made using differences of least squares means with a Bonferroni adjustment when significant $F$ test values from the ANOVA were obtained. SAS 9.2 (TS2M3) software was used for all analyses. Outlier values were removed (FUS1:Wt-5 min C, Wt-360 min B, GMTcp-272-5 min A, GMTcp-272-30 min C; FVEG_00078-Wt-360 min B, $\Delta$ SGE$360 \mathrm{~min}$ B, and GMTcp-272-5 min A) so that $\log$ transformations could stabilize variances for all four genes before ANOVAs could be run.

\section{Analysis of SM.}

Fumonisins and fusarins were extracted from $2.5 \mathrm{~g}$ of cracked maize kernel cultures with $12 \mathrm{ml}$ of acetonitrile/water (84:16, vol/vol). Cultures were allowed to steep for $2 \mathrm{~h}$, with gentle shaking, and then extracts were filtered through a $0.45-\mu \mathrm{m}$-poresize Gelman filter. The culture extracts were then analyzed for fumonisin $\mathrm{FB}_{1}, \mathrm{FB}_{2}$, and $\mathrm{FB}_{3}$ and fusarin $\mathrm{C}$ by LC-MS/MS, essentially as previously described (Brown et al. 2012; Plattner et al. 1996). In brief, the analytical method utilized a LCMS/MS instrument consisting of a ThermoSpetraPhysics highperformance liquid chromatography (HPLC) system and a ThermoFinnigan LCQ DECA ion trap mass spectrometer. The mass spectrometer was operated in positive mode utilizing an electrospray ionization (ESI) interface. Injections of $10 \mu \mathrm{l}$ of analyte were eluted from a Metachem (Torrance, CA, U.S.A.) Inertsil $\mathrm{C} 183$ by $150 \mathrm{~mm}$ column with a $300 \mu \mathrm{l} / \mathrm{min}$ gradient flow of water/methanol $(\mathrm{MeOH})$. The entire column flow was directed to the ESI interface of the mass spectrometer. HPLC solvents were acidified with $0.3 \%$ acetic acid. The gradient program consisted of the following steps: 0 to $10 \mathrm{~min}, 35 \% \mathrm{MeOH}$; 10 to $25 \mathrm{~min}, 35$ to $95 \% \mathrm{MeOH}$; 25 to $26 \mathrm{~min}, 95 \% \mathrm{MeOH} ; 26$ to $34 \mathrm{~min}, 95$ to $35 \% \mathrm{MeOH}$; and 34 to $35 \mathrm{~min}, 35 \% \mathrm{MeOH}$. ESI-MS/MS detection of fusarin $\mathrm{C}$ was accomplished by monitoring three characteristic fragment ions ( $\mathrm{m} / \mathrm{z} 364,382$, and 396) of the $\mathrm{m} / \mathrm{z} 432[\mathrm{M}+\mathrm{H}]+$ ion of fusarin $\mathrm{C}$ in multiple reaction monitoring mode. Quantization of fusarin $C$ was done on the basis of the integrated intensity of the $\mathrm{m} / \mathrm{z} 364$ fragment compared with a calibration curve generated from fusarin $\mathrm{C}$ standard solutions. Signal for LC-MS/MS determination of fusarin $\mathrm{C}$ may contain multiple products from the decomposition of fusarin $\mathrm{C}$ during the LC-MS analysis. Fusaric acid was extracted from cracked maize cultures with $12 \mathrm{ml}$ of acetonitrile/water (50:50, vol/vol) acidified to $\mathrm{pH} 2$ with $\mathrm{HCl}$ and analyzed by LCMS/MS. Extract analysis was accomplished essentially as described (Brown et al. 2012). Fusaric acid standard was obtained from Sigma-Aldrich.

\section{ACKNOWLEDGMENTS}

We thank C. McGovern, C. Probyn, and M. Moore for technical assistance and D. Palmquist for assistance with the statistical analysis.

\section{LITERATURE CITED}

Allen, A., Snyder, A. K., Preuss, M., Nielsen, E. E., Shah, D. M., and Smith, T. J. 2008. Plant defensins and virally encoded fungal toxin KP4 inhibit plant root growth. Planta 227:331-339. 
Altschul, S. F., Madden, T. L., Schaffer, A. A., Zhang, J., Zhang, Z., Miller, W., and Lipman, D. J. 1997. Gapped BLAST and PSI-BLAST: A new generation of protein database search programs. Nucleic Acids Res. 25:3389-3402.

Bacon, C. W., Yates, I. E., Hinton, D. M., and Meredith, F. 2001. Biological control of Fusarium moniliforme in maize. Environ. Health Perspect. 109:325-332.

Bai, G. H., Desjardins, A. E., and Plattner, R. D. 2002. Deoxynivalenolnonproducing Fusarium graminearum causes initial infection, but does not cause disease spread in wheat spikes. Mycopathologia 153:91-98.

Bluhm, B. H., Kim, H., Butchko, R. A., and Woloshuk, C. P. 2008. Involvement of ZFRI of Fusarium verticillioides in kernel colonization and the regulation of FST1, a putative sugar transporter gene required for fumonisin biosynthesis on maize kernels. Mol. Plant Pathol. 9:203211.

Bolstad, B. M., Irizarry, R. A., Astrand, M., and Speed, T. P. 2003. A comparison of normalization methods for high density oligonucleotide array data based on variance and bias. Bioinformatics 19:185-193.

Brown, D. W. 2011. The KP4 killer protein gene family. Curr. Genet. 57:51-62.

Brown, D. W., Butchko, R. A., Busman, M., and Proctor, R. H. 2007. The Fusarium verticillioides FUM gene cluster encodes a $\mathrm{Zn}$ (II)2Cys6 protein that affects $F U M$ gene expression and fumonisin production. Eukaryot. Cell. 6:1210-1218.

Brown, D. W., McCormick, S. P., Alexander, N. J., Proctor, R. H., and Desjardins, A. E. 2002. Inactivation of a cytochrome P-450 is a determinant of trichothecene diversity in Fusarium species. Fungal Genet. Biol. 36:224-233.

Brown, D. W., Cheung, F., Proctor, R. H., Butchko, R. A., Zheng, L., Lee, Y., Utterback, T., Smith, S., Feldblyum, T., Glenn, A. E., Plattner, R. D., Kendra, D. F., Town, C. D., and Whitelaw, C. A. 2005. Comparative analysis of 87,000 expressed sequence tags from the fumonisin-producing fungus Fusarium verticillioides. Fungal Genet. Biol. 42:848-861.

Brown, D. W., Butchko, R. A., Busman, M., and Proctor, R. H. 2012. Identification of gene clusters associated with fusaric acid, fusarin, and perithecial pigment production in Fusarium verticillioides. Fungal Genet. Biol. 49:521-532.

Burgess, L. W. 1981. General ecology of the Fusaria. Pages 225-235 in: Fusarium: Diseases, Biology, and Taxonomy. P. E. Nelson, T. A Toussoun, and R. J. Cook, eds. The Pennsylvania State University Press, University Park.

Butchko, R. A., Plattner, R. D., and Proctor, R. H. 2003. FUM9 is required for C-5 hydroxylation of fumonisins and complements the meitotically defined Fum3 locus in Gibberella moniliformis. Appl. Environ. Microbiol. 69:6935-6937.

Catlett, N. L., Lee, B. N., Yoder, O. C., and Turgeon, B. G. 2002. Splitmarker recombination for efficient targeted deletion of fungal genes. Fungal. Genet. News 49:9-11.

Claing, A., Laporte, S. A., Caron, M. G., and Lefkowitz, R. J. 2002. Endocytosis of $\mathrm{G}$ protein-coupled receptors: Roles of $\mathrm{G}$ protein-coupled receptor kinases and beta-arrestin proteins. Prog. Neurobiol. 66:61-79.

de Jonge, R., Bolton, M. D., and Thomma, B. P. 2011. How filamentous pathogens co-opt plants: The ins and outs of fungal effectors. Curr Opin. Plant Biol. 14:400-406.

Desjardins, A. E., and Proctor, R. H. 2007. Molecular biology of Fusarium mycotoxins. Int. J. Food Microbiol. 119:47-50.

Desjardins, A. E., Munkvold, G. P., Plattner, R. D., and Proctor, R. H. 2002. FUM1-a gene required for fumonisin biosynthesis but not for maize ear rot and ear infection by Gibberella moniliformis in field tests. Mol. Plant-Microbe Interact. 15:1157-1164.

Desjardins, A. E., Busman, M., Muhitch, M., and Proctor, R. H. 2007. Complementary host-pathogen genetic analyses of the role of fumonisins in the Zea mays-Gibberella moniliformis interaction. Physiol. Mol. Plant Pathol. 70:149-160.

DeZwaan, T. M., Carroll, A. M., Valent, B., and Sweigard, J. A. 1999. Magnaporthe grisea Pth $11 \mathrm{p}$ is a novel plasma membrane protein that mediates appressorium differentiation in response to inductive substrate cues. Plant Cell 11:2013-2030.

Fernandes, M., Keller, N. P., and Adams, T. H. 1998. Sequence-specific binding by Aspergillus nidulans AflR, a C6 zinc cluster protein regulating mycotoxin biosynthesis. Mol. Microbiol. 28:1355-1365.

Fuchs, U., Czymmek, K. J., and Sweigard, J. A. 2004. Five hydrophobin genes in Fusarium verticillioides include two required for microconidial chain formation. Fungal Genet. Biol. 41:852-864.

Gage, M. J., Rane, S. G., Hockerman, G. H., and Smith, T. J. 2002. The virally encoded fungal toxin KP4 specifically blocks L-type voltagegated calcium channels. Mol. Pharmacol. 61:936-944.

Glenn, A. E. 2007. Mycotoxigenic Fusarium species in animal feed. Anim. Feed Sci. Technol. 137:213-240.

Glenn, A. E., Zitomer, N. C., Zimeri, A. M., Williams, L. D., Riley, R. T., and Proctor, R. H. 2008. Transformation-mediated complementation of a FUM gene cluster deletion in Fusarium verticillioides restores both fumonisin production and pathogenicity on maize seedlings. Mol. Plant-Microbe Interact. 21:87-97.

Houterman, P. M., Speijer, D., Dekker, H. L., CG, D. E. K., Cornelissen, B. J., and Rep, M. 2007. The mixed xylem sap proteome of Fusarium oxysporum-infected tomato plants. Mol. Plant Pathol. 8:215-221.

Houterman, P. M., Ma, L., van Ooijen, G., de Vroomen, M. J., Cornelissen, B. J., Takken, F. L., and Rep, M. 2009. The effector protein Avr2 of the xylem-colonizing fungus Fusarium oxysporum activates the tomato resistance protein I-2 intracellularly. Plant J. 58:970-978.

Huang, G., Wang, H., Chou, S., Nie, X., Chen, J., and Liu, H. 2006. Bistable expression of WORl, a master regulator of white-opaque switching in Candida albicans. Proc. Natl. Acad. Sci. U.S.A. 103:12813-12818.

Irizarry, R. A., Hobbs, B., Collin, F., Beazer-Barclay, Y. D., Antonellis, K. J., Scherf, U., and Speed, T. P. 2003. Exploration, normalization, and summaries of high density oligonucleotide array probe level data. Biostatistics 4:249-264.

Jonkers, W., Dong, Y., Broz, K., and Kistler, H. C. 2012. The Wor1-like protein Fgp1 regulates pathogenicity, toxin synthesis and reproduction in the phytopathogenic fungus Fusarium graminearum. PLoS Pathog. $8: 1-18$.

Kim, K. H., Cho, Y., M, L. A. R., Cramer, R. A., Jr., and Lawrence, C. B. 2007. Functional analysis of the Alternaria brassicicola non-ribosomal peptide synthetase gene AbNPS2 reveals a role in conidial cell wall construction. Mol. Plant Pathol. 8:23-39.

Larson, T. M., Kendra, D. F., Busman, M., and Brown, D. W. 2011. Fusarium verticillioides chitin synthases $\mathrm{CHS} 5$ and $\mathrm{CHS} 7$ are required for normal growth and pathogenicity. Curr. Genet. 57:177-189.

Leslie, J. F., Plattner, R. D., Desjardins, A. E., and Klittich, C. J. R. 1992. Fumonisin $\mathrm{B}_{1}$ production by strains from different mating populations of Gibberella fujikuroi (Fusarium section Liseola). Phytopathology 82:341-345.

Ma, L. J., van der Does, H. C., Borkovich, K. A., Coleman, J. J., Daboussi, M. J., Di Pietro, A., Dufresne, M., Freitag, M., Grabherr, M., Henrissat, B., Houterman, P. M., Kang, S., Shim, W. B., Woloshuk, C., Xie, X., Xu, J. R., Antoniw, J., Baker, S. E., Bluhm, B. H., Breakspear, A., Brown, D. W., Butchko, R. A. E., Chapman, S., Coulson, R., Coutinho, P. M., Danchin, E. G., Diener, A., Gale, L. R., Gardiner, D. M., Goff, S. Hammond-Kosack, K. E., Hilburn, K., Hua-Van, A., Jonkers, W., Kazan, K., Kodira, C. D., Koehrsen, M., Kumar, L., Lee, Y. H., Li, L., Manners, J. M., Miranda-Saavedra, D., Mukherjee, M., Park, G., Park, J., Park, S. Y., Proctor, R. H., Regev, A., Ruiz-Roldan, M. C., Sain, D., Sakthikumar, S., Sykes, S., Schwartz, D. C., Turgeon, B. G., Wapinski, I., Yoder, O., Young, S., Zeng, Q., Zhou, S., Galagan, J., Cuomo, C. A., Kistler, H. C., and Rep, M. 2010. Comparative genomics reveals mobile pathogenicity chromosomes in Fusarium oxysporum. Nature 464:367373.

Marasas, W. F., Riley, R. T., Hendricks, K. A., Stevens, V. L., Sadler, T. W., Gelineau-Van Waes, J., Missmer, S. A., Cabrera, J., Torres, O., Gelderblom, W. C., Allegood, J., Martinez, C., Maddox, J., Miller, J. D., Starr, L., Sullards, M. C., Roman, A. V., Voss, K. A., Wang, E., and Merrill, A. H., Jr. 2004. Fumonisins disrupt sphingolipid metabolism, folate transport, and neural tube development in embryo culture and in vivo: A potential risk factor for human neural tube defects among populations consuming fumonisin-contaminated maize. J. Nutr. 134:711716.

Michielse, C. B., van Wijk, R., Reijnen, L., Cornelissen, B. J., and Rep, M. 2009a. Insight into the molecular requirements for pathogenicity of Fusarium oxysporum f. sp. lycopersici through large-scale insertional mutagenesis. Genome Biol. 10:1-18.

Michielse, C. B., van Wijk, R., Reijnen, L., Manders, E. M., Boas, S. Olivain, C., Alabouvette, C., and Rep, M. 2009b. The nuclear protein Sge1 of Fusarium oxysporum is required for parasitic growth. PLoS Pathog. 5:e1000637.

Michielse, C. B., Becker, M., Heller, J., Moraga, J., Collado, I. G., and Tudzynski, P. 2011. The Botrytis cinerea Reg1 protein, a putative transcriptional regulator, is required for pathogenicity, conidiogenesis, and the production of secondary metabolites. Mol. Plant-Microbe Interact. 24:1074-1085.

Morgavi, D. P., and Riley, R. T. 2007. An historical overview of field disease outbreaks known or suspected to be caused by consumption of feeds contaminated with Fusarium toxins. Anim. Feed Sci. Technol. 137:201-212.

Murillo, I., Cavallarin, L., and Segundo, B. S. 1999. Cytology of infection of maize seedlings by Fusarium moniliforme and immunolocalization of the pathogenesis-related PRms protein. Phytopathology 89:737-747.

Myung, K., Zitomer, N. C., Duvall, M., Glenn, A., Riley, R. T., and Calvo, A. M. 2011. The conserved global regulator VeA is necessary for symptom production and mycotoxin synthesis in maize seedlings by Fusarium 
verticillioides. Plant Pathol. 16:152-160.

Nelson, P. E. 1992. Taxonomy and biology of Fusarium moniliforme. Mycopathologia 117:29-36.

Nghiem, N. P., Montanti, J., Johnston, D. B., and Drapcho, C. 2011. Fractionation of corn fiber treated by soaking in aqueous ammonia (SAA) for isolation of hemicellulose B and production of C5 sugars by enzyme hydrolysis. Appl. Biochem. Biotechnol. 164:1390-1404.

Nguyen, V. Q., and Sil, A. 2008. Temperature-induced switch to the pathogenic yeast form of Histoplasma capsulatum requires Ryp1, a conserved transcriptional regulator. Proc. Natl. Acad. Sci. U.S.A. 105:4880-4885.

Niehaus, E. M., Kleigrewe, K., Wiemann, P., Studt, L., Sieber, C. M., Connolly, L. R., Freitag, M., Guldener, U., Tudzynski, B., and Humpf, H. U. 2013. Genetic manipulation of the Fusarium fujikuroi fusarin gene cluster yields insight into the complex regulation and fusarin biosynthetic pathway. Chem. Biol. 20:1055-1066.

Oren, L., Ezrati, S., Cohen, D., and Sharon, A. 2003. Early events in the Fusarium verticillioides-maize interaction characterized by using a green fluorescent protein-expressing transgenic isolate. Appl. Environ. Microbiol. 69:1695-1701

Plattner, R. D., Wiesleder, D., and Poling, S. M. 1996. Analytical determination of fumonisins and other metabolites produced by Fusarium moniliforme and related species on corn. Pages 57-64 in: Alkaloids: Chemical and Biochemical Perspectives. L. S. Jackson, J. W. De Vries, and L. B. Bullerman, eds. Pergamon Press, Elmsford, NY, U.S.A.

Rafiqi, M., Ellis, J. G., Ludowici, V. A., Hardham, A. R., and Dodds, P. N. 2012. Challenges and progress towards understanding the role of effectors in plant-fungal interactions. Curr. Opin. Plant Biol. 15:477-482.

Rep, M., Dekker, H. L., Vossen, J. H., de Boer, A. D., Houterman, P. M. Speijer, D., Back, J. W., de Koster, C. G., and Cornelissen, B. J. 2002. Mass spectrometric identification of isoforms of PR proteins in xylem sap of fungus-infected tomato. Plant Physiol. 130:904-917.

Santhanam, P., and Thomma, B. P. 2013. Verticillium dahliae Sge1 differentially regulates expression of candidate effector genes. Mol. PlantMicrobe Interact. 26:249-256.

Seong, K. Y., Pasquali, M., Zhou, X., Song, J., Hilburn, K., McCormick, S., Dong, Y., Xu, J. R., and Kistler, H. C. 2009. Global gene regulation by Fusarium transcription factors Tri6 and Tri10 reveals adaptations for toxin biosynthesis. Mol. Microbiol. 72:354-367.

Takken, F., and Rep, M. 2010. The arms race between tomato and Fusarium oxysporum. Mol. Plant Pathol. 11:309-314.

Tuite, J. 1969. Plant Pathological Methods: Fungi and Bacteria. Burgess Publishing Company, Minneapolis, MN, U.S.A.

Turgeon, B. G., Garber, R. C., and Yoder, O. C. 1987. Development of a fungal transformation system based on selection of sequences with promoter activity. Mol. Cell. Biol. 7:3297-3305. van der Does, H. C., Duyvesteijn, R. G., Goltstein, P. M., van Schie, C. C Manders, E. M., Cornelissen, B. J., and Rep, M. 2008. Expression of effector gene SIXI of Fusarium oxysporum requires living plant cells. Fungal Genet. Biol. 45:1257-1264.

Wang, C., and St Leger, R. J. 2007. The MAD1 adhesin of Metarhizium anisopliae links adhesion with blastospore production and virulence to insects, and the MAD2 adhesin enables attachment to plants. Eukaryot. Cell. 6:808-816.

Wiemann, P., Sieber, C. M., von Bargen, K. W., Studt, L., Niehaus, E. M., Espino, J. J., Huss, K., Michielse, C. B., Albermann, S., Wagner, D., Bergner, S. V., Connolly, L. R., Fischer, A., Reuter, G., Kleigrewe, K. Bald, T., Wingfield, B. D., Ophir, R., Freeman, S., Hippler, M., Smith, K. M., Brown, D. W., Proctor, R. H., Munsterkotter, M., Freitag, M. Humpf, H. U., Guldener, U., and Tudzynski, B. 2013. Deciphering the cryptic genome: Genome-wide analyses of the rice pathogen Fusarium fujikuroi reveal complex regulation of secondary metabolism and novel metabolites. PLoS Pathog. 9:e1003475.

Woloshuk, C. P., and Shim, W. B. 2013. Aflatoxins, fumonisins, and trichothecenes: A convergence of knowledge. FEMS (Fed. Eur. Microbiol. Soc.) Microbiol. Rev. 37:94-109.

Wong, K. H., Hynes, M. J., Todd, R. B., and Davis, M. A. 2009. Deletion and overexpression of the Aspergillus nidulans GATA factor AreB reveals unexpected pleiotropy. Microbiology 155:3868-3880.

Wu, F. 2007. Measuring the economic impacts of Fusarium toxins in animal feeds. Anim. Feed Sci. Technol. 137:363-374.

Zadra, I., Abt, B., Parson, W., and Haas, H. 2000. xylP promoter-based expression system and its use for antisense downregulation of the Penicillium chrysogenum nitrogen regulator NRE. Appl. Environ. Microbiol. 66:4810-4816.

Zhang, Z., Schaffer, A. A., Miller, W., Madden, T. L., Lipman, D. J., Koonin, E. V., and Altschul, S. F. 1998. Protein sequence similarity searches using patterns as seeds. Nucleic Acids Res. 26:3986-3990.

Zordan, R. E., Miller, M. G., Galgoczy, D. J., Tuch, B. B., and Johnson, A. D. 2007. Interlocking transcriptional feedback loops control whiteopaque switching in Candida albicans. PLoS Biol. 5:e256.

\section{AUTHOR-RECOMMENDED INTERNET RESOURCES}

Fusarium Comparative database: www.broadinstitute.org/annotation/genome/fusarium_group/MultiHome. html

Dana-Farber Cancer Institute-Harvard School of Public Health $F$. verticillioides Gene Index database: compbio.dfci.harvard.edu/tgi/cgi-bin/tgi/gimain.pl?gudb=f_verticill 